\title{
Comparative investigation of ELM control based on toroidal modelling of plasma response to RMP fields
}

\author{
Yueqiang Liu, ${ }^{1,2,3, a), b)}$ A. Kirk, ${ }^{1}$ Li Li, ${ }^{4, c)}$ Y. In, ${ }^{5}$ R. Nazikian, ${ }^{6}$ Youwen Sun, ${ }^{7}$ W. Suttrop, ${ }^{8}$ \\ B. Lyons, ${ }^{9}$ D. Ryan, ${ }^{10,1}$ Shuo Wang, ${ }^{2}$ Xu Yang, ${ }^{11}$ Lina Zhou, ${ }^{11}$ \\ and EUROfusion MST1 team ${ }^{\text {d) }}$ \\ ${ }^{1}$ CCFE, Culham Science Centre, Abingdon OX14 3DB, United Kingdom \\ ${ }^{2}$ Southwestern Institute of Physics, P.O. Box 432, Chengdu 610041, China \\ ${ }^{3}$ Department of Earth and Space Science, Chalmers University of Technology, SE-41296 Gothenburg, Sweden \\ ${ }^{4}$ Donghua University, Shanghai, China \\ ${ }^{5}$ National Fusion Research Institute, KSTAR Research Center 169-148, Daejeon, South Korea \\ ${ }^{6}$ Princeton Plasma Physics Laboratory, Princeton University, P.O. Box 451, Princeton, \\ New Jersey 08543-0451, USA \\ ${ }^{7}$ Institute of Plasma Physics, Chinese Academy of Sciences, P.O. Box 1126, Hefei 230031, China \\ ${ }^{8}$ Max Planck Institute for Plasma Physics, Garching, Germany \\ ${ }^{9}$ General Atomics, P.O. Box 85608, San Diego, California 92186-5608, USA \\ ${ }^{10}$ Department of Physics, University of York, Heslington, York YO10 5DD, United Kingdom \\ ${ }^{11}$ Dalian University of Technology, Dalian 116024, China
}

(Received 11 November 2016; accepted 23 February 2017; published online 22 March 2017)

Extensive modelling efforts of the plasma response to the resonant magnetic perturbation fields, utilized for controlling the edge localized mode (ELM), help to identify the edge-peeling response as a key factor, which correlates to the observed ELM mitigation in several tokamak devices, including MAST, ASDEX Upgrade, EAST, and HL-2A. The recently observed edge safety factor window for ELM mitigation in HL-2A experiments is explained in terms of the edge-peeling response. The computed plasma response, based on toroidal single fluid resistive plasma model with different assumption of toroidal flows, is found generally larger in ELM suppressed cases as compared to that of the ELM mitigated cases, in ASDEX Upgrade and DIII-D. The plasma shaping, in particular, the plasma triangularity, contributes to the enhanced plasma response. But the shaping does not appear to be the sole factor-other factors such as the (higher) pedestal pressure and/or current can also lead to increased edge-peeling response.

[http://dx.doi.org/10.1063/1.4978884]

\section{INTRODUCTION}

Resonant magnetic perturbation (RMP), or sometimes simply referred to as magnetic perturbation (MP), has been experimentally established as an efficient way of controlling the large (type-I) edge localized mode (ELM) in H-mode tokamak plasmas. In fact, full suppression of type-I ELM, under ITER relevant (low) collisionality conditions, has been reported on several present-day devices, including DIII-D, ${ }^{1}$ $\mathrm{KSTAR}^{2}{ }^{2} \mathrm{EAST},{ }^{3}$ and ASDEX Upgrade. ${ }^{4}$ Even in devices where ELM suppression has so far not been achieved, the ELM bursting frequency is significantly increased with reduced amplitude per burst. This is referred to as ELM mitigation, which is still of significant benefit in terms of reducing the peak heat flux load on the plasma facing components. ELM mitigation (but not suppression) has been achieved in $\mathrm{JET},{ }^{5} \mathrm{MAST},{ }^{6}$ and very recently in HL-2A.

Extensive modelling efforts have been devoted to understand and to interpret ELM control experiments. Most of the modelling work has been carried out for individual devices, for example, MAST, ${ }^{7}$ DIII-D, ${ }^{8,9}$ ASDEX Upgrade, ${ }^{10-13}$

\footnotetext{
Note: Paper BI2 3, Bull. Am. Phys. Soc. 61, 21 (2016).

a) Invited speaker.

b)E-mail: yueqiang.liu@ukaea.uk

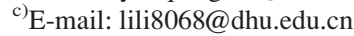

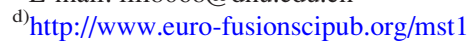

EAST,${ }^{14}$ and ITER. ${ }^{7,15-17}$ These activities were also reported in recent review articles. ${ }^{18,19}$

In this work, we report some of the recent multimachine, comparative modelling results on the ELM control experiments utilizing the RMP fields. More specifically, we focus on the toroidal computation of the plasma response to RMP fields. The plasma response, in terms of either the perturbed magnetic field or the plasma displacement, often allows a direct comparison with experimental measurements and consequently quantitative validation of the computational models. ${ }^{20,21}$ In turn, the plasma response can provide, at the macroscopic level, guidance for optimization of the coil configuration in order to achieve the best ELM control in experiments. No less importantly, the plasma response computations also help to understand the RMP field penetration physics and the associated particle and momentum transport. ${ }^{22,23}$ Finally, the computed plasma response field can be valuable input data for further studies, such as the (enhanced) energetic particle losses due to 3D RMP fields, and the resulting divertor-wall heat loads. ${ }^{24}$

This study has revealed that there are essentially two types of plasma response to the applied RMP fields: One is the so-called core-kink response and the other is the edgepeeling response. ${ }^{7}$ The difference is that the plasma displacement is strongly localised near the plasma edge-in the pedestal region-with the edge-peeling type of response and is 
often associated with a dominant single poloidal harmonics in terms of the radial plasma displacement. The core-kink response, on the contrary, has a much more global structure in terms of the internal plasma displacement. The corresponding poloidal spectrum of the radial displacement is also richer.

It has been computationally observed that the core-kink response normally results in a large plasma surface displacement near the outboard mid-plane, similar to the ballooning mode structure, though in this case with rather low toroidal mode numbers ( $n$ is typically equal to $1,2,3$ ). On the other hand, the edge-peeling response is often associated with a large plasma displacement near the X-point. For a resistive plasma response, the amplitude of the resonant radial magnetic field components near the plasma edge is also found to be a good measure for the edge-peeling type of plasma response.

From the practical point of view, perhaps the most useful result is the correlation between the computed edgepeeling response and the observed ELM mitigation by the RMP fields in experiments. Such a correlation has previously been obtained in the modelling of several tokamak devices ${ }^{4,7,8,13,25}$ and will be further confirmed by the new results presented in this work. The role of the edge-peeling response in ELM suppression is less exploited in the previous work and is therefore one of the focusing points in this study.

Section II briefly introduces the computational model that we use in this study to obtain the plasma response in toroidal geometry. Section III presents the multi-machine modelling results for the ELM mitigation experiments, followed by the ELM suppression modelling reported in Section IV. Section V summarizes the computational results, and Section VI draws conclusion.

\section{COMPUTATIONAL PLASMA RESPONSE MODEL}

The majority of the results reported in this study are obtained by the MARS-F code ${ }^{26}$ which solves linearized, single fluid MHD equations in toroidal geometry. The detailed formulation for solving the RMP problem was described in Ref. 27, with additional comments on certain specific points being also discussed in a recent work. ${ }^{11}$

For the completeness of information, below we list the equations that are solved by MARS-F

$$
\begin{gathered}
i\left(\Omega_{\mathrm{RMP}}+n \Omega\right) \xi=\mathbf{v}+(\xi \cdot \nabla \Omega) R \hat{\phi}, \\
i \rho\left(\Omega_{\mathrm{RMP}}+n \Omega\right) \mathbf{v}=-\nabla p+\mathbf{j} \times \mathbf{B}+\mathbf{J} \times \mathbf{b} \\
-\rho[2 \Omega \hat{\mathbf{Z}} \times \mathbf{v}+(\mathbf{v} \cdot \nabla \Omega) R \hat{\phi}] \\
-\rho \kappa_{\|}\left|k_{\|} v_{t h, i}\right|\left[\mathbf{v}+(\xi \cdot \nabla) \mathbf{V}_{0}\right]_{\|}, \\
i\left(\Omega_{\mathrm{RMP}}+n \Omega\right) \mathbf{b}=\nabla \times(\mathbf{v} \times \mathbf{B})+(\mathbf{b} \cdot \nabla \Omega) R \hat{\phi}-\nabla \times(\eta \mathbf{j}), \\
i\left(\Omega_{\mathrm{RMP}}+n \Omega\right) p=-\mathbf{v} \cdot \nabla P-\Gamma P \nabla \cdot \mathbf{v}, \\
\mathbf{j}=\nabla \times \mathbf{b},
\end{gathered}
$$

where $R$ is the plasma major radius, $\hat{\phi}$ the unit vector along the geometric toroidal angle $\phi$ of the torus, and $\hat{\mathbf{Z}}$ the unit vector in the vertical direction in the poloidal plane. $\Omega_{\mathrm{RMP}}$ is the excitation frequency of the RMP field, which is zero for a dc coil current. $n$ is the toroidal harmonic number. For a linear response of axi-symmetric equilibria, we need to consider only a single $n$ perturbation each time. The full plasma response from different $n$ s can in principle be linearly superposed, if needed. The plasma resistivity is denoted by $\eta$. The Spitzer resistivity model is used in this work. The variables $\xi, \mathbf{v}, \mathbf{b}, \mathbf{j}$, and $p$ represent the plasma displacement, perturbed velocity, magnetic field, current, and pressure, respectively. The equilibrium plasma density, field, current, and pressure are denoted by $\rho, \mathbf{B}, \mathbf{J}$, and $P$, respectively. $\Gamma=5 / 3$ is the ratio of specific heats for ideal gas.

The last term in Eq. (2) describes the effect of parallel sound wave damping, with $\kappa$ being a numerical coefficient determining the damping "strength." $k_{\|}=(n-m / q) / R$ is the parallel wave number, with $m$ being the poloidal harmonic number and $q$ being the safety factor. $v_{t h, i}=$ $\sqrt{2 T_{i} / M_{i}}$ is the thermal ion velocity, with $T_{i}$ and $M_{i}$ being the thermal ion temperature and mass, respectively. The parallel component of the perturbed velocity is taken along the equilibrium field line. In this work, we assume $\kappa_{\|}=1.5$, corresponding to a strong sound wave damping. The influence of the sound wave damping model (strong versus weak) has been systematically investigated in Ref. 11 .

The RMP field is generated by the source current $\mathbf{j}_{\text {RMP }}$ flowing in the RMP coils

$$
\nabla \times \mathbf{b}=\mathbf{j}_{\mathrm{RMP}}, \quad \nabla \cdot \mathbf{j}_{\mathrm{RMP}}=0 .
$$

Note that MARS-F consistently solves the combined MHD equations in the plasma region, the vacuum equations for the perturbed field b (i.e., curl- and divergence free conditions for b) outside the plasma, as well as the coil equation (6) as the source term. The perturbed magnetic field $\mathbf{b}$ is thus defined as a global quantity across the plasma-vacuum-coil regions. In other words, the b field defined in MHD equations as well as in Eq. (6) is produced by the currents both in the plasma (the perturbed plasma current) and in the RMP coils.

The two key physics terms in our model, which are directly relevant to the plasma response to the RMP fields, are the toroidal flow frequency $\Omega$ and the plasma resistivity $\eta$. The former leads to the screening of the applied vacuum field (more precisely the resonant components), while the latter allows certain penetration of the field. Within the linear theory, the superposition of the resistive plasma response field and the $2 \mathrm{D}$ equilibrium field yields magnetic islands of finite size, as a result of "forced reconnection."

Within the single fluid theory, the plasma flow is naturally presented by the thermal ion flow $\Omega$. However, since the major effect of the flow is the field shielding in the RMP plasma response problem, we shall also consider another flow model, namely, the equilibrium $\mathbf{E} \times \mathbf{B}$ flow, with the rotation frequency of $\Omega_{\mathrm{E} \times \mathrm{B}}$, in this work. In other words, in some of the study reported in Section IV, we shall replace $\Omega$ from the above MHD equations by $\Omega_{\mathrm{E} \times \mathrm{B}}$ and compare the plasma response with these two different flow models. Different flow models certainly correspond to different MHD physics. 
However, the mathematical structure corresponding to the field screening, which is reflected in the Ohm's law, is very similar. $^{28}$ We also mention that, within the two-fluid theory, the electron flow in the direction perpendicular to the magnetic field line has been shown to be crucial for the resonant field screening. ${ }^{29-31}$ This flow model is beyond the physics capability of our present formulation and is thus not considered here.

Despite the relative simplicity of the formulation, the linear single fluid model has been shown to be quantitatively adequate in many cases, in particular, for the RMP problem associated with the ELM control, when the modelling results are compared with the experimental measurements. ${ }^{20,21}$

\section{MODELLING ELM MITIGATION EXPERIMENTS}

ELM mitigation has been achieved in several machines, including MAST, 6,32 ASDEX Upgrade, ${ }^{33}$ and $\mathrm{JET}^{5}$ and recently in $\mathrm{EAST}^{3}$ and HL-2A. Extensive MARS-F modelling has been performed for MAST, ${ }^{7}$ ASDEX Upgrade, ${ }^{10-12}$ and EAST. ${ }^{14}$ All the modelling results, in comparison with the corresponding experimental observations, so far point to the important role played by the edge-peeling response for achieving the best ELM mitigation. ${ }^{19}$ The edge-peeling response, which was first identified in modelling of the JET experiments, ${ }^{34}$ is found to be closely correlated to the pronounced plasma surface displacement near the X-point. On the contrary, the other type of the plasma response- the core-kink response-often causes large plasma displacement near the outboard mid-plane, due to the ballooning effect. In the modelling of the MAST ELM mitigation experiments, we found that the ratio of the plasma displacement near the $\mathrm{X}$-point to that at the outboard mid-plane serves as a good indicator for the density pump-out observed in experiments, for both $\mathrm{L}$ - and H-mode plasmas. In H-mode plasmas, achieving ELM mitigation without causing the mode locking or the H-to-L back transition requires this displacement ratio exceeding a certain critical value (about 1.7 for MAST plasmas). In other words, the best strategy for ELM mitigation appears to be maximizing the edge-peeling response and at the same time minimizing the core-kink response.

For the purpose of avoiding confusion with terminology, we briefly explain here the meaning of the edge-peeling response, which has been discussed in several of previous studies. This is one type of kink response, which causes the plasma displacement mainly near the edge, and is thus sometimes also referred to as the "edge-kink" response in literatures. The structure of the perturbation is similar to that of the peeling mode instability, with the latter being (normally the low- $n$ ) part of the spectrum of the peeling-ballooning mode, which is the initial MHD instability associated with type-I ELMs. The difference is that the peeling mode normally refers to an unstable eigenmode, whilst the edgepeeling response refers to part of the stable plasma response to the RMP fields. The toroidal spectrum of the peelingballooning mode eventually depends on the plasma equilibrium, whilst the edge-peeling response always has the same toroidal spectrum as that of the applied vacuum RMP field.
In ASDEX Upgrade plasmas using the $n=2$ RMP coil configurations, the plasma flow is often partially damped during the ELM mitigated phase, but without directly causing mode locking. Extensive modelling efforts, performed for discharges with the conventional plasma shape (low upper triangularity), again reveal the importance of the edgepeeling response. In particular, the fluid model predicted optimal coil phasing, which maximizes the edge-peeling response, agrees well with the best achievable ELM mitigation in experiments. ${ }^{33}$ This is confirmed by MARS-F, ${ }^{10-12}$ NEMEC, ${ }^{4}$ and JOREK ${ }^{13}$ computations. In particular, systematic scans with varying edge safety factor ${ }^{12,37}$ as well as plasma pressure ${ }^{37}$ yield simple analytic fitting formulas for the optimal coil phasing, with varying plasma conditions.

ELM mitigation has also been achieved in EAST with the $n=2$ RMP fields. The computational study, reported in Ref. 14, again reveals the important role played by the edgepeeling response. More specifically, coil phasing of $+90^{\circ}$ and $-90^{\circ}$ was considered in experiments. The $+90^{\circ}$ phase, though introducing much larger resonant field components compared to the $-90^{\circ}$ phase, has a very weak effect on the ELM behavior. The $-90^{\circ}$ coil phasing is computationally shown to cause large edge-peeling response and experimentally strong ELM mitigation.

In the following, we shall report the MARS-F modelling results for the recent ELM mitigation experiments in HL-2A. HL-2A is a medium-sized tokamak with the major radius of $R_{0}=1.65 \mathrm{~m}$ and the typical plasma minor radius of about $37 \mathrm{~cm}$. A $2 \times 2$ ELM control coil system has recently been installed. There are two rows of coils (upper and lower, respectively) as shown in Fig. 1, with each consisting of 2 coils along the toroidal angle $\phi$, spanning about $11.4^{\circ}$ in $\phi$, and being separated from each other by $180^{\circ}$ in $\phi$.

This coil system generates multiple toroidal RMP field components. By supplying the coil currents flowing in the opposite direction in each row, as in experiments, field components with odd $n$ numbers are created. In HL-2A discharge 29676, which we use in this work for the modelling purpose, the supplied coil current is $4.5 \mathrm{kAt}$. An analytic estimate shows that the corresponding coil currents for toroidal components $n=1,3,5$, and 7 are $284 \mathrm{~A}, 280 \mathrm{~A}, 373 \mathrm{~A}$, and $262 \mathrm{~A}$, respectively. The amplitude of the first few toroidal components of the coil currents is comparable, as expected.

However, when the generated RMP fields reach the plasma, the (resonant) field components with higher $n$ become significantly weaker, due to the fact that the higher$n$ and $m$ ( $m$ is the poloidal number) components decay faster in the vacuum. The resulting resonant radial field amplitude, at the corresponding rational surface close to the plasma boundary, is compared in Fig. 2. Here, the amplitude of the radial field component, for each $m$ and $n$, is defined as a dimensionless quantity

$$
b_{\mathrm{res}}^{1} \equiv \frac{1}{R_{0}^{2} B_{0}}\left|\frac{\mathbf{b} \cdot \nabla \psi}{\mathbf{B}_{\mathrm{eq}} \cdot \nabla \phi}\right|_{m n},
$$

where $B_{0}$ is the vacuum toroidal magnetic field strength at the major radius $R_{0}\left(B_{0}=1.37\right.$ Tesla in HL-2A discharge 29676), b the perturbed magnetic field due to RMP, $\psi$ the 

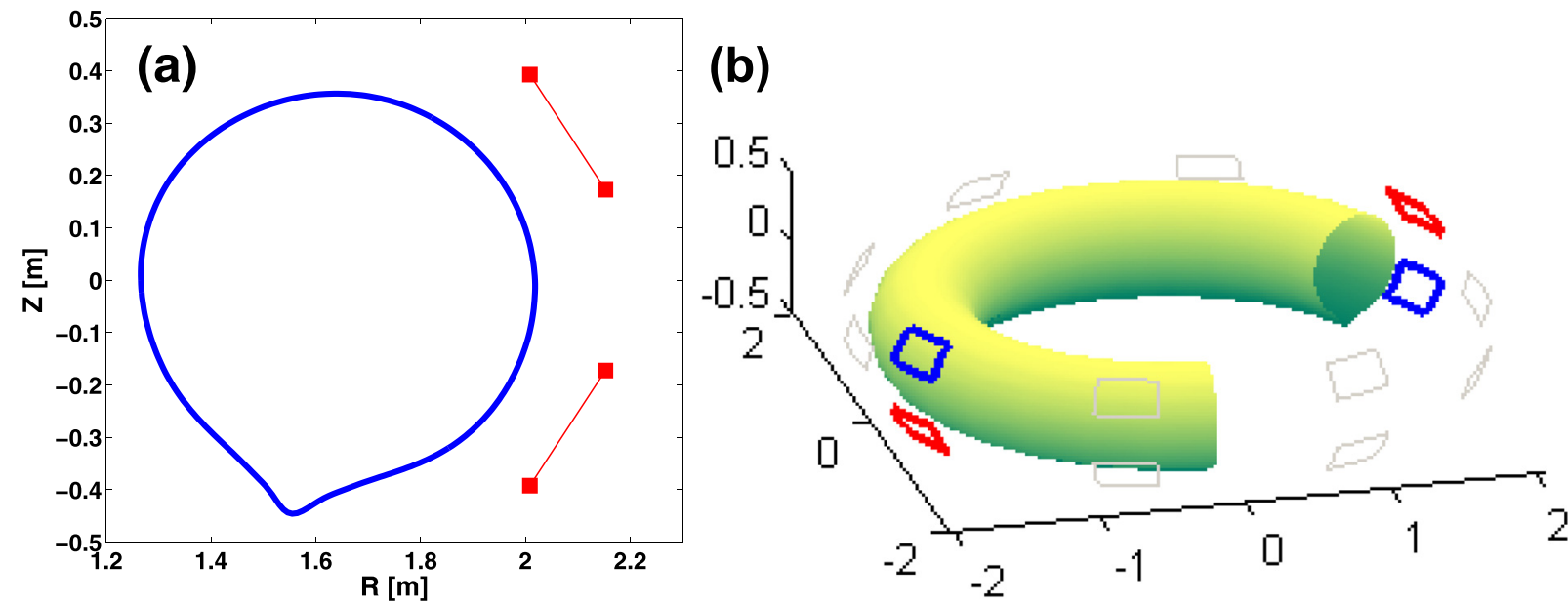

FIG. 1. The location and size of the ELM control coils in HL-2A, shown (a) on the poloidal plane together with the plasma boundary shape for discharge 29676 at $820 \mathrm{~ms}$, and (b) in a 3D view in blue and red.

equilibrium poloidal flux function, and $\mathbf{B}_{\mathrm{eq}}$ the equilibrium field. The toroidal harmonic is calculated using the geometric toroidal angle $\phi$, whilst the poloidal harmonic is calculated using a PEST-like definition for the poloidal angle $\chi$, which yields a Jacobian being proportional to the square of
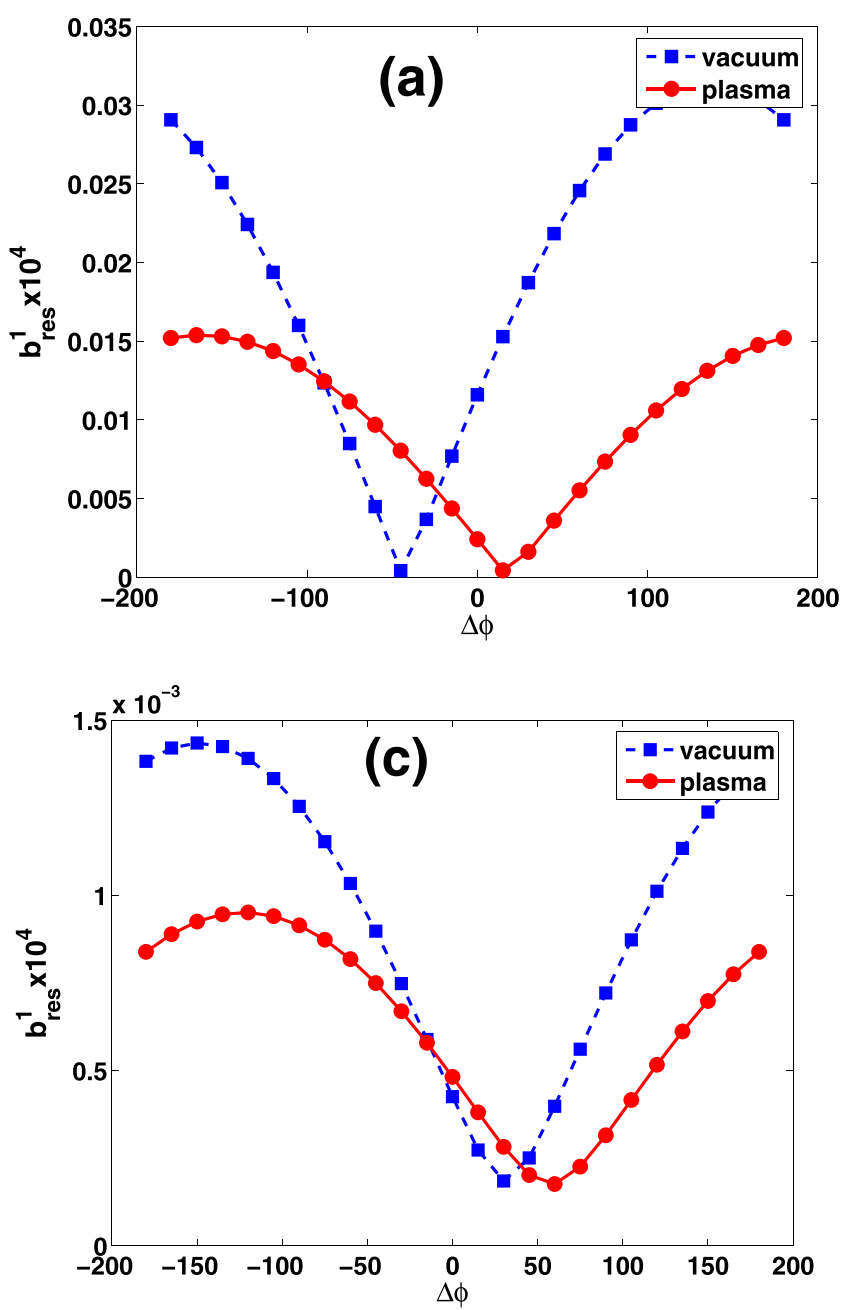

major radius, $R^{2}$. These choices of toroidal and poloidal angles result in a straight-field-line flux coordinate system.

Figure 2 compares $b_{\text {res }}^{1}$ for $n=1,3,5,7$ toroidal components. For each $n$, comparison is also made between the applied vacuum RMP field (dashed lines) and the total
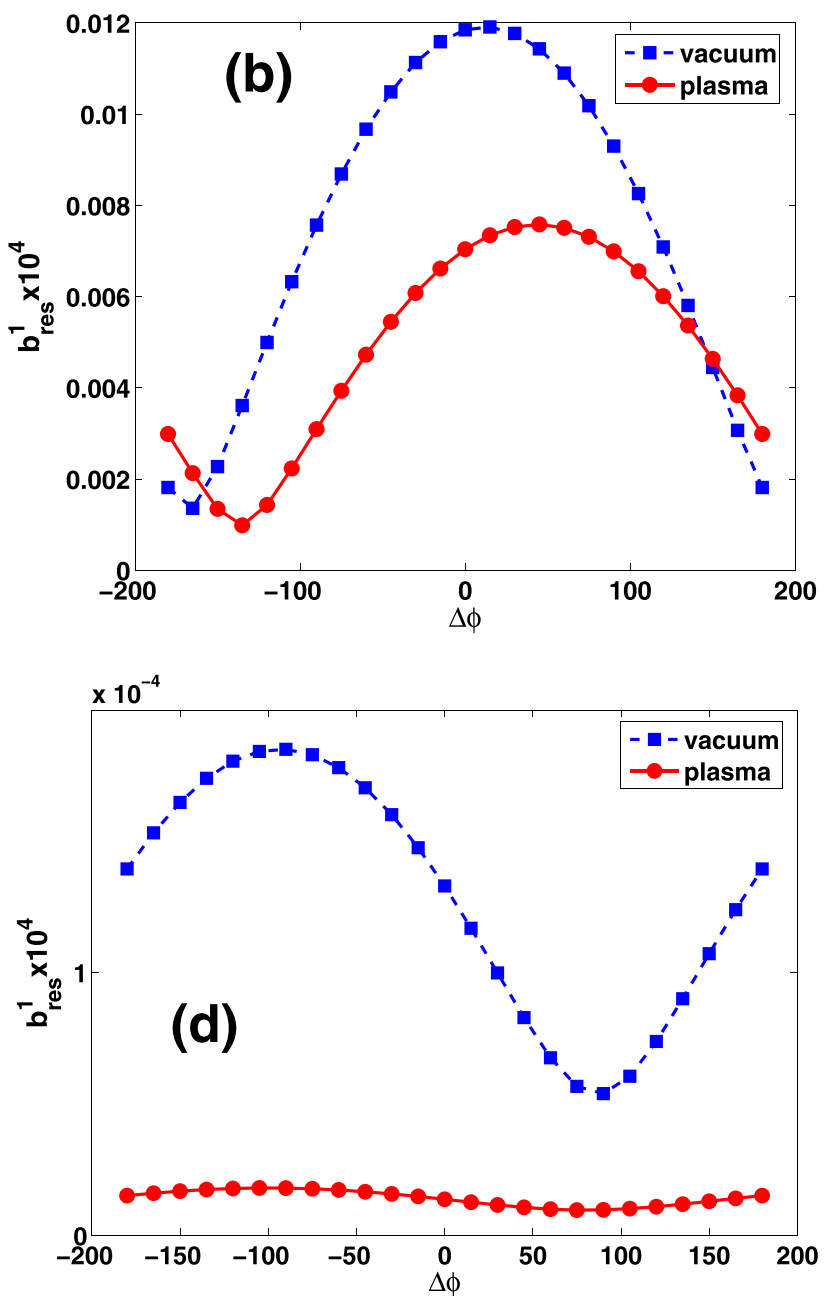

FIG. 2. The computed amplitude of the last resonant radial field component, for the vacuum RMP field (dashed lines) and the total response field including the plasma response (solid lines), for the (a) $n=1$, (b) $n=3$, (c) $n=5$, and (d) $n=7$ field components in HL-2A, with artificial variation of the coil phasing $\Delta \phi$ between the upper and lower rows. The experimental coil phasing corresponds to $\Delta \phi=180^{\circ}$. 
perturbed field, including the plasma response (solid lines). Moreover, in numerical modelling, for each $n$, we perform full scans of the coil current phasing angle $\Delta \phi$ between the upper and lower rows, from $-180^{\circ}$ to $+180^{\circ}$. In experiments, with only two coils per row, the only possible choice for the coil phasing is either even parity $(\Delta \phi=0)$ or odd parity $\left(\Delta \phi= \pm 180^{\circ}\right)$. In all ELM control experiments carried out so far in HL-2A, only odd parity configuration has been considered.

Comparing the resonant vacuum field components between $n=1,3,5,7$, we find that the ratio of the peak values (among all coil phasing $\Delta \phi$ ) is about 169:64:8:1, indicating that the largest role in ELM control is still played by the $n=1$ field component in HL-2A, despite a very small coverage of the toroidal angle by the RMP coils. The $n=3$ field component, being 3 times smaller than the $n=1$ component, may also play some role.

The inclusion of the plasma response changes the poloidal spectrum of the RMP field. As a consequence, the dependence of $b_{\text {res }}^{1}$ on the coil phasing $\Delta \phi$ also changes. In particular, the peak amplitude of $b_{\text {res }}^{1}$ is reached at different coil phasing, between the vacuum field and the total response field. Defining the coil phasing that maximizes $b_{\text {res }}^{1}$ as the "optimal" coil phasing, we find $60^{\circ}$ shift in the optimal phasing between the total response field and the vacuum field, for the dominant $n=1$ component. This $60^{\circ}$ phase shift is close to what has been found for ASDEX Upgrade plasmas ${ }^{10-12}$ as well.

More interestingly, the $b_{\text {res }}^{1}$ value from the $n=1$ plasma response peaks at $\Delta \phi=180^{\circ}$, i.e., with the odd parity coil configuration, indicating that the experimental choice of the coil configuration is already optimal. The other possible choice of the coil configuration in experiments-the even parity - should yield the least effect on ELMs, according to the modelling results shown here.

As has been shown in the previous MARS-F modeling for MAST, ${ }^{7}$ ASDEX Upgrade, ${ }^{10-12}$ and EAST, ${ }^{14}$ ELM mitigation is closely correlated to the edge-peeling response, which often manifests itself as pronounced plasma displacement near the X-point. ${ }^{19} \mathrm{We}$ examine here these aspects for the HL-2A case, with results summarized in Figs. 3 and 4, where we again scan the coil phasing $\Delta \phi$. Figure 3 compares the amplitude of the core-kink component (dashed lines) versus the edge-peeling component (solid lines) of the plasma
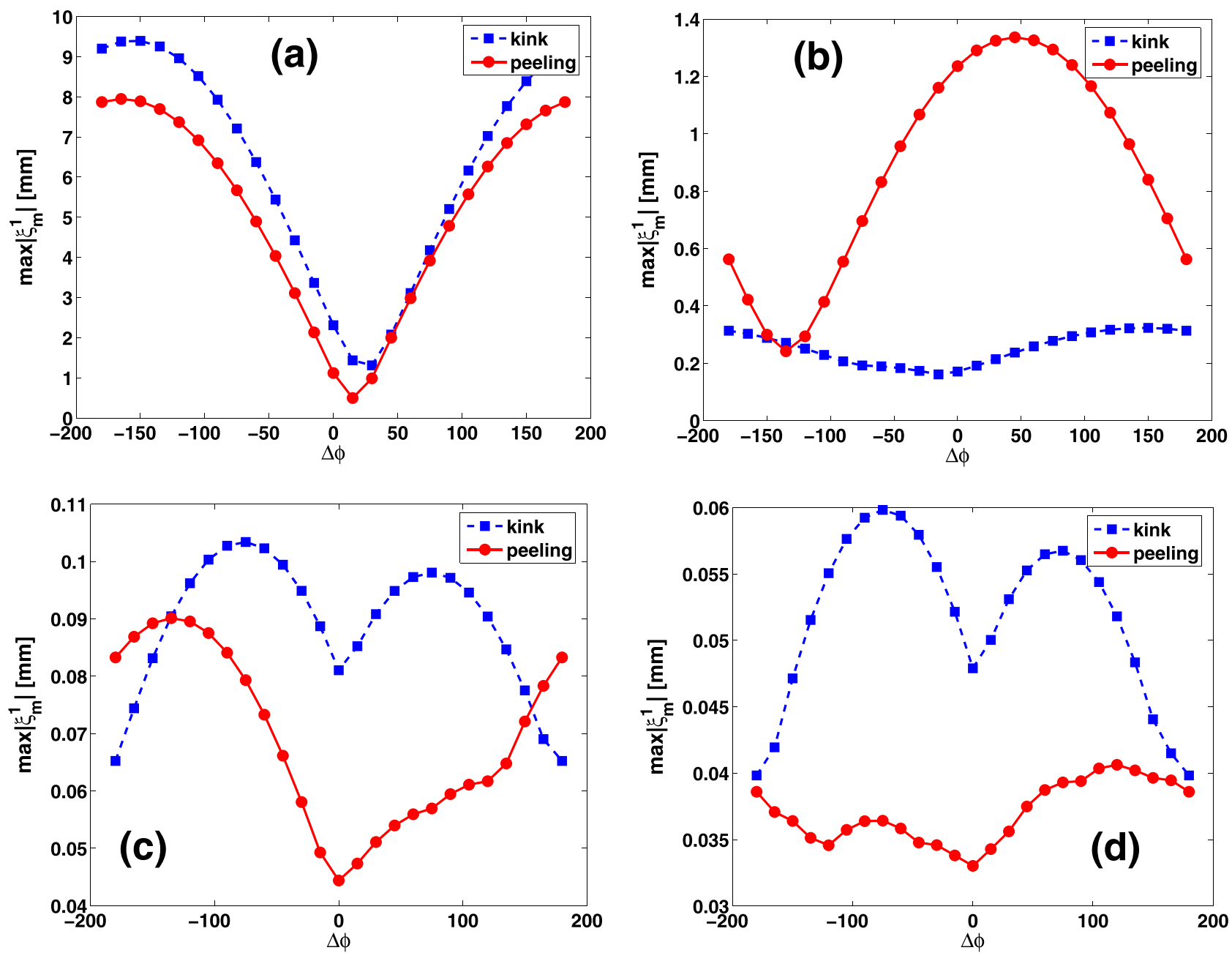

FIG. 3. The computed amplitude of the core-kink (dashed lines) versus the edge-peeling (solid lines) components of the plasma response, caused by the (a) $n=1$, (b) $n=3$, (c) $n=5$, and (d) $n=7$ vacuum RMP field components in HL-2A, with artificial variation of the coil phasing $\Delta \phi$ between the upper and lower rows. The experimental coil phasing corresponds to $\Delta \phi=180^{\circ}$. The amplitude of the response components is measured in terms of the radial plasma displacement. 

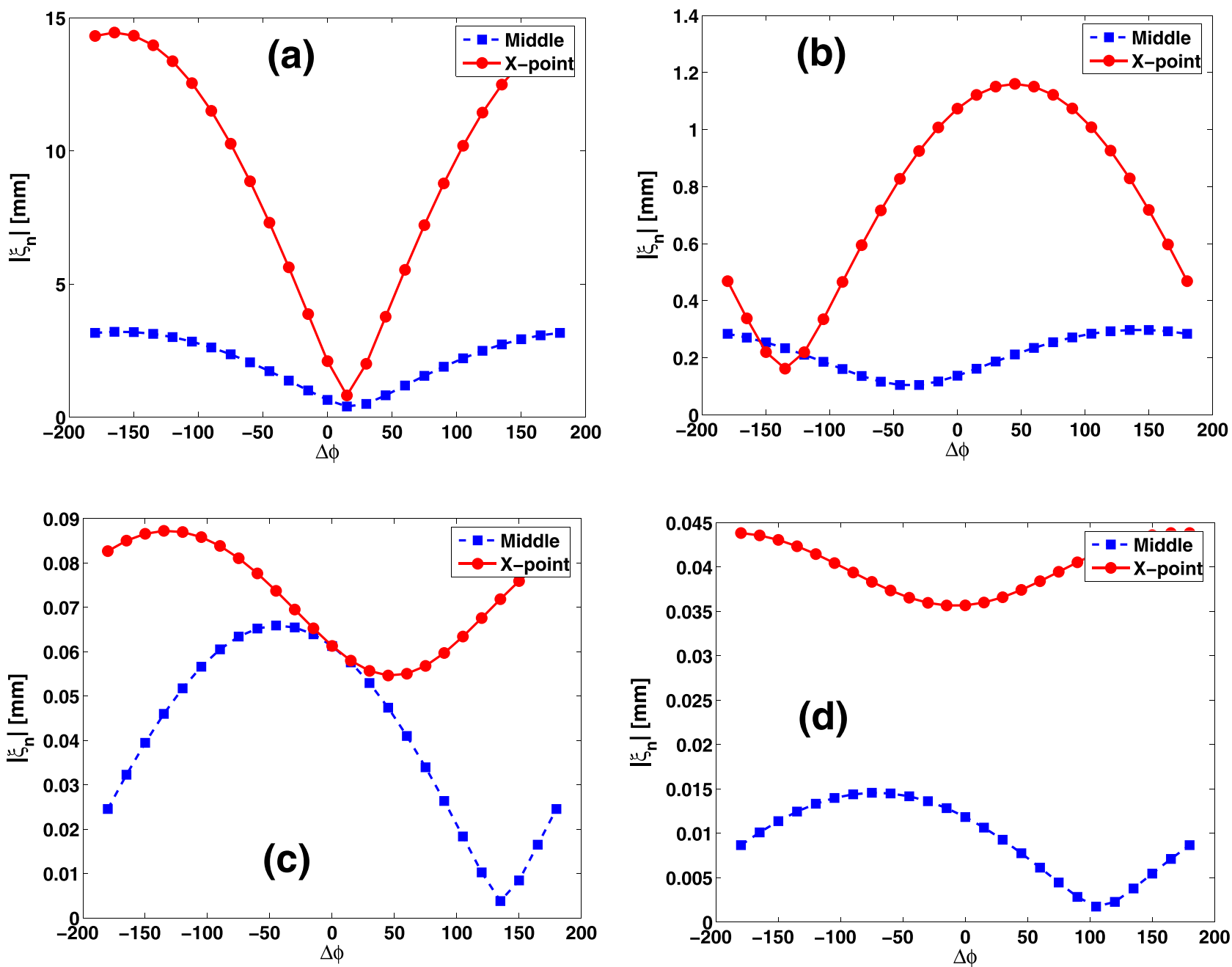

FIG. 4. The computed amplitude of the plasma surface displacement near the outboard mid-plane (dashed lines) and near the X-point (solid lines), caused by the (a) $n=1$, (b) $n=3$, (c) $n=5$, and (d) $n=7$ vacuum RMP field components in HL-2A, with artificial variation of the coil phasing $\Delta \phi$ between the upper and lower rows. The experimental coil phasing corresponds to $\Delta \phi=180^{\circ}$.

response, for the $n=1,3,5,7$ RMP fields, respectively. The amplitude of the core-kink response is defined as the maximum value of all poloidal Fourier harmonics of the computed plasma radial displacement $\left|\xi_{m n}^{1}\left(\psi_{p}\right)\right| \equiv|\xi \cdot \nabla s|_{m n}$, in the range of the normalized equilibrium poloidal flux $0<\psi_{p} \equiv s^{2}<0.5$ (i.e., in the plasma core region). The amplitude of the edge-peeling response is defined as the maximum amplitude of the same quantity in the range of $0.8<\psi_{p}<1$ (i.e., in the plasma edge regions). Although these definitions are not unique, the previous modelling experience shows that this choice well represents the core-kink and the edgepeeling components of the plasma response.

Two key observations can be made from Fig. 3. First, the plasma radial displacement quickly decreases with the toroidal mode number. In particular, for the edge-peeling amplitude, the peak values (along $\Delta \phi$ ) follow a ratio of 196:33:2:1, for $n=1,3,5,7$. This is generally consistent with the resonant radial field amplitude ratio shown in Fig. 2, again confirming the dominant role played by the $n=1$ RMP field component for the ELM control in HL-2A. The second observation is that, for the $n=1$ harmonic, the edge-peeling amplitude reaches the maximum value at $\Delta \phi \sim 180^{\circ}$-another indication that the odd parity coil configuration is close to the optimum for the ELM control in HL-2A. It is also interesting to note that the core-kink amplitude also reaches maximum with the odd parity coil configuration, for this HL-2A plasma.

Closely related to the edge-peeling (core-kink) response is the plasma surface displacement near the X-point (the outboard mid-plane). This is indeed confirmed by the computed coil phasing scan results as shown in Fig. 4. Here, $\xi_{n} \equiv$ $|\xi \cdot \nabla s| /|\nabla s|$ is the amplitude of the normal displacement of the plasma surface. The correlation is particularly evident for the dominant toroidal components $n=1$ and 3. Less correlation is observed for $n=5$ and 7. But the amplitude of the plasma displacement is very small for $n=5$ and 7 . The good correlation between the edge-peeling response and the plasma displacement near the X-point, for $n=1,3$, also means that the odd parity coil configuration causes the largest X-point displacement in these HL-2A plasmas.

The amplitude of the plasma normal displacement is also plotted in the poloidal plane in Fig. 5, and compared between the $n=1$ and the $n=3$ toroidal components, assuming the odd parity coil configuration. Besides the obvious difference in the overall magnitude of the displacement, the pattern is somewhat different. In particular, the $n=1$ normal displacement strongly peaks near the X-point, whilst the 

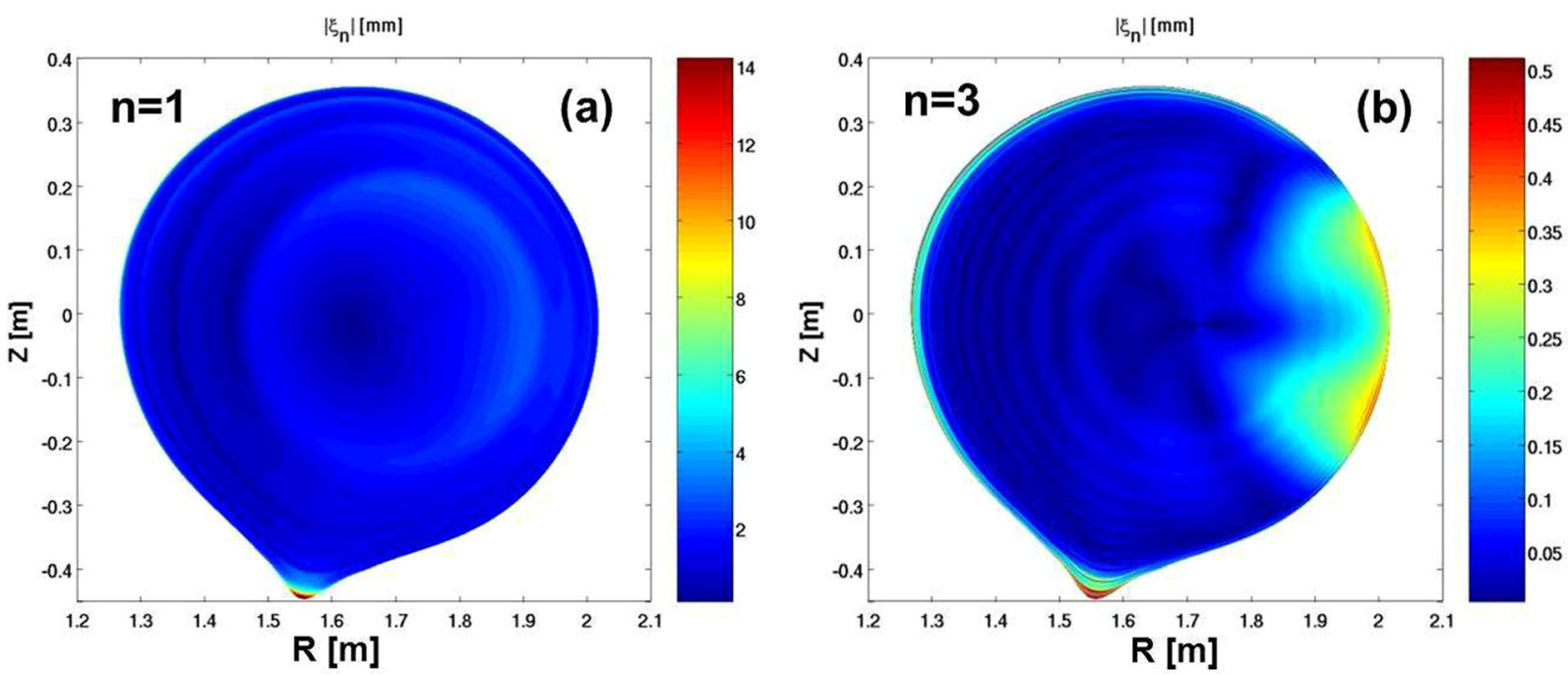

FIG. 5. The computed distribution of the plasma radial displacement amplitude at the poloidal plane, caused by the (a) $n=1$ and (b) $n=3$ components of the applied RMP fields in HL-2A. The ELM control coil current is assumed to be $4.5 \mathrm{kAt}$, with the upper and lower rows in odd parity, as in experiments.

$n=3$ displacement is pronounced both near the X-point and in the low field side region of the torus. The combined effect is still largely the X-point displacement peaking with the odd parity coils, which should be in favour of maximizing the control effects on the ELMs in experiments.
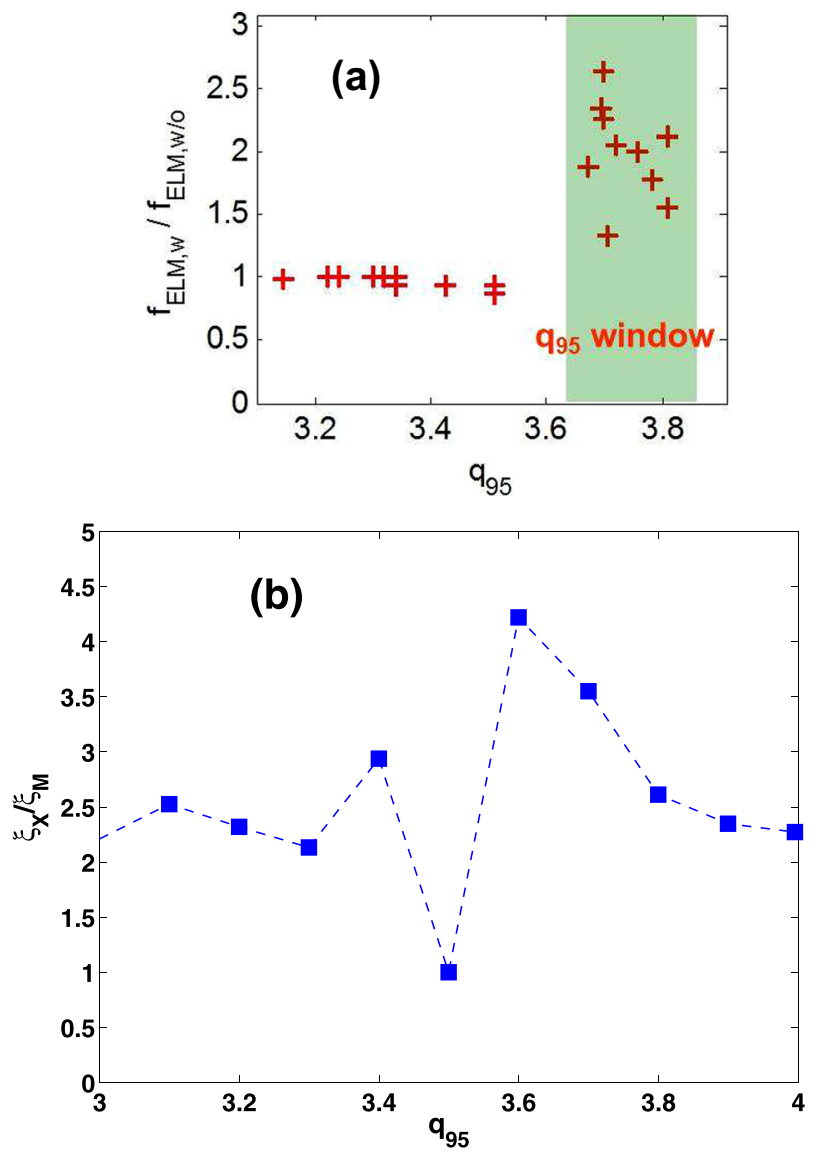

FIG. 6. Comparison of the HL-2A experiments versus the modelling results as the safety factor $q_{95}$ is scanned: (a) the ratio of the ELM frequency with RMP to that without RMP, as measured in experiments, (b) the ratio of the plasma surface displacement near the X-point to that near the outboard midplane, as computed by MARS-F.
Finally, and perhaps most interestingly, is a direct comparison between experiments and modelling for HL-2A, as shown in Fig. 6. Here, we present in Fig. 6(a) the experimentally measured ELM frequency for a series of RMP discharges, where the edge safety factor $q_{95}$ is varied. The ELM frequency is normalized by that from the RMP-off discharges. In the range of $q_{95}$ below 3.5, no ELM mitigation is achieved in HL-2A (with odd parity coil configuration). However, clear ELM mitigation is achieved in a $q_{95}$ window with $q_{95}$ value above 3.6, with more than doubling of the ELM frequency in certain cases.

In the MARS-F modelling results shown in Fig. 6(b), we vary $q_{95}$ by scanning the total plasma current, based on the plasma equilibrium from the HL-2A discharge 29676 at $820 \mathrm{~ms}$. We find that, roughly in the same $q_{95}$ window where the ELM mitigation has been observed in experiments, the ratio $\xi_{X} / \xi_{M}$ of the computed plasma surface displacement near the X-point, to that of the outboard mid-plane, is maximized. This is qualitatively the same correlation we found for the MAST plasmas. ${ }^{7}$ On the other hand, we notice that the correlation is not perfect, between the ELM mitigation window obtained in experiments (Fig. 6(a)) and that from the modelling (Fig. 6(b)). In particular, the modelling predicts a mitigation window, which is slightly shifted towards the lower range of $q_{95}$. This may be partially due to the way the plasma equilibria are scanned in MARS-F, where only the total plasma current is varied, without modifying other equilibrium quantities such as the current profile and the

TABLE I. Basic equilibrium parameters of the modelled ASDEX Upgrade (discharge numbers 30835 and 33133) and DIII-D (discharge number 164277) plasmas.

\begin{tabular}{lcccccccc}
\hline \hline Shot\# & Time $(\mathrm{ms})$ & $R_{0}(\mathrm{~m})$ & $B_{0}(\mathrm{~T})$ & $I_{p}(\mathrm{MA})$ & $\beta_{N}$ & $q_{0}$ & $q_{95}$ & $\Omega_{0} / \omega_{A}(\%)$ \\
\hline 30835 & 3200 & 1.724 & 1.705 & 0.773 & 2.148 & 0.811 & 3.760 & 2.299 \\
33133 & 3000 & 1.701 & 1.756 & 0.854 & 2.064 & 1.129 & 3.782 & 4.551 \\
164277 & 2500 & 1.670 & 1.907 & 1.587 & 1.665 & 1.157 & 3.640 & 5.081 \\
\hline
\end{tabular}



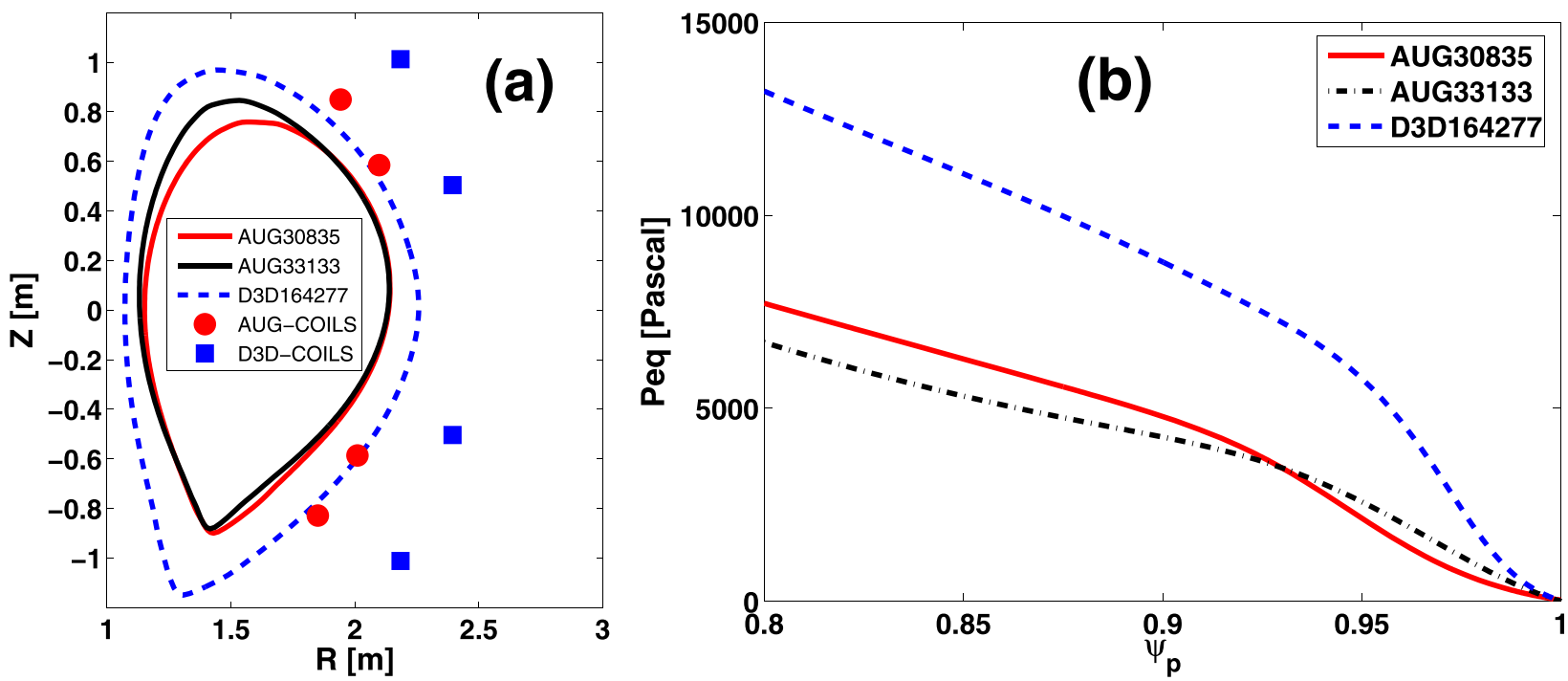

FIG. 7. Comparison of (a) the plasma boundary shapes and the ELM control coils location between the ASDEX Upgrade discharge 30835 (with ELM mitigation) and 33133 (ELM suppression), and the DIII-D discharge 164277 (ELM suppression), and (b) the equilibrium pressure profiles near the plasma edge (covering the pedestal region) among three discharges. The equilibria are reconstructed within an inter-ELM period during the ELM mitigated phase for the ASDEX Upgrade discharge 30835, and during the ELM suppressed phase for ASDEX Upgrade 33133 and DIII-D 164277 .

plasma pressure. In experiments, these quantities may vary from shots to shots. Nevertheless, these MARS-F modelling results for HL-2A, though still not representing an exhausted study, already confirm the role of the edge-peeling response in the ELM mitigation that we have previously found in other devices.

\section{MODELLING ELM SUPPRESSION EXPERIMENTS}

So far, most of our modelling efforts have been devoted to the ELM mitigation experiments. Work has just been started to compute the plasma response for ELM suppressed experiments. In particular, understanding the physics

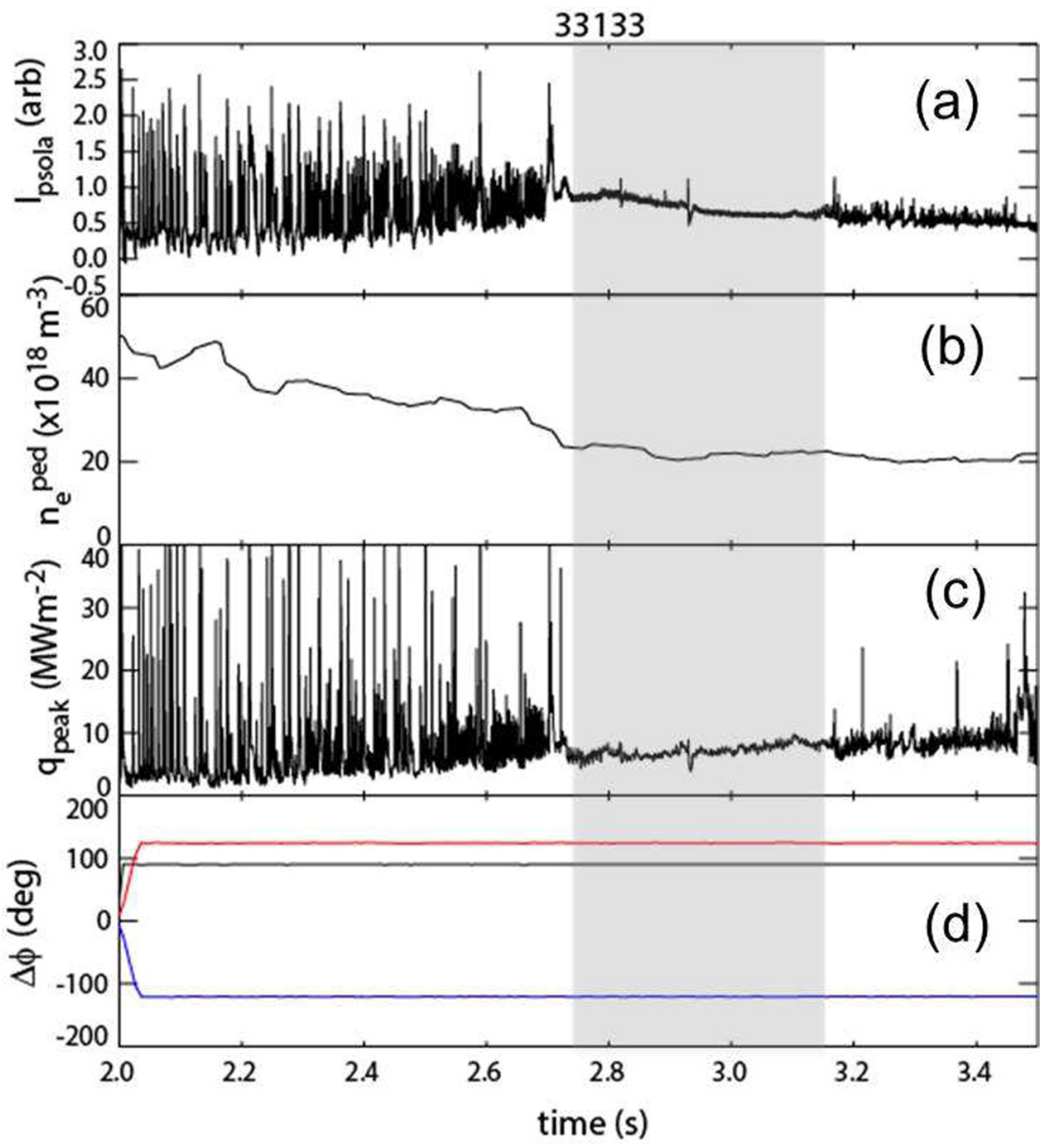

FIG. 8. Time traces for the ELM suppressed discharge 33133 in ASDEX Upgrade, for (a) the divertor current (the outer divertor thermoelectric current), (b) the pedestal density, (c) the divertor peak heat flux, and (d) the coil current phasing $\left(90^{\circ}\right.$ in this case). 
difference between the ELM mitigation and suppression is still at the initial stage, with some of the results reported below for ASDEX Upgrade, DIII-D, and KSTAR.

Before doing so, we briefly mention the previous modelling efforts for the ELM suppression experiments in DIII$\mathrm{D}^{25}$ and EAST. ${ }^{14}$ Despite the possible profound difference between the mitigation and suppression physics, both studies still found that the edge-peeling response is an important indicator for the ELM suppression. In particular, a systematic coil phasing scan for the EAST plasma confirms that the best coil phasing for achieving the ELM suppression is the one that causes the strongest edge-peeling response. ${ }^{14}$

In the following, we report a comparative analysis of the plasma response computed by MARS-F, for both ELM mitigation and suppression discharges. Full ELM suppression has recently been achieved in ASDEX Upgrade under low pedestal collisionality conditions (with effective electron collisionality at the pedestal top $\left.\nu_{\text {ped }}^{*} \leq 0.4\right){ }^{4}$ This was possible, however, only with the increased plasma shaping. More specifically, it was found that increasing the upper triangularity helps to obtain the ELM suppression. In the conventional, low upper triangularity plasmas, only ELM mitigation was achieved, even with the optimal ELM control coil configurations. ${ }^{33}$ It is therefore important to understand, from the plasma response point of view, whether (and how) a stronger plasma shaping helps to achieve the ELM suppression.

For this purpose, we select three plasmas from three ELM control experiments-two from ASDEX Upgrade and one from DIII-D. These three plasmas, with key equilibrium parameters listed in Table I, differ significantly in the plasma shaping, in particular, the upper triangularity, as shown in Fig. 7(a). The conventional low triangularity ASDEX Upgrade plasma, represented by discharge 30835 , has upper triangularity $\delta_{U}=0.05$ and lower triangularity $\delta_{L}=0.43$. The high triangularity ASDEX Upgrade shape, represented by discharge 33133, has $\delta_{U}=0.23$ and $\delta_{L}=0.42$. Finally, the DIII-D discharge 164277 has ITER similar shape (ISS), with $\delta_{U}=0.34$ and $\delta_{L}=0.65$. We note that these three equilibria have a similar edge safety factor of $q_{95} \simeq 3$.7. The pedestal pressure is significantly higher in DIII-D discharge 164277, as shown in Fig. 7(b). The pedestal pressure in ASDEX Upgrade discharge 33133 is slightly higher than that of 30835 .

In experiments, ELM mitigation is achieved in discharges similar to ASDEX Upgrade 30835, using the $n=2$ RMP fields produced by $5 \mathrm{kAt}$ coil currents in $90^{\circ}$ coil phasing, which is close to the optimal coil phasing as judged by the edge-peeling response criterion. ${ }^{11,12}$ Using the same coil configuration, however, ELM suppression is achieved in ASDEX Upgrade discharge 33133, as shown in Fig. 8 using the same coil phasing and 6.5 kAt RMP coil currents. Indeed, both the divertor current (a) and the divertor heat flux (c) measurements show a full ELM suppression in the time window of $2.75-3.15 \mathrm{~s}$, at a fixed coil phasing of $90^{\circ}$ (d). The plasma pedestal density decreases (density pump out) after the application of the RMP fields, but remains nearly constant during the ELM suppression phase. In DIII-D discharge 164277, a 4.5 kAt coil current, with the $n=3$ even parity configuration, is sufficient to suppress the type-I ELM.
In this work, we shall compute and compare the plasma response for the aforementioned three equilibria, using the corresponding coil configurations as in experiments. A key input parameter for the plasma response computation is the plasma flow. Within the single fluid model in MARS-F, we shall test two toroidal flow models. One is the fluid flow model (i.e., the bulk thermal ion flow), with the toroidal angular rotation frequency of $\Omega$. The other is the $\mathbf{E} \times \mathbf{B}$ flow,
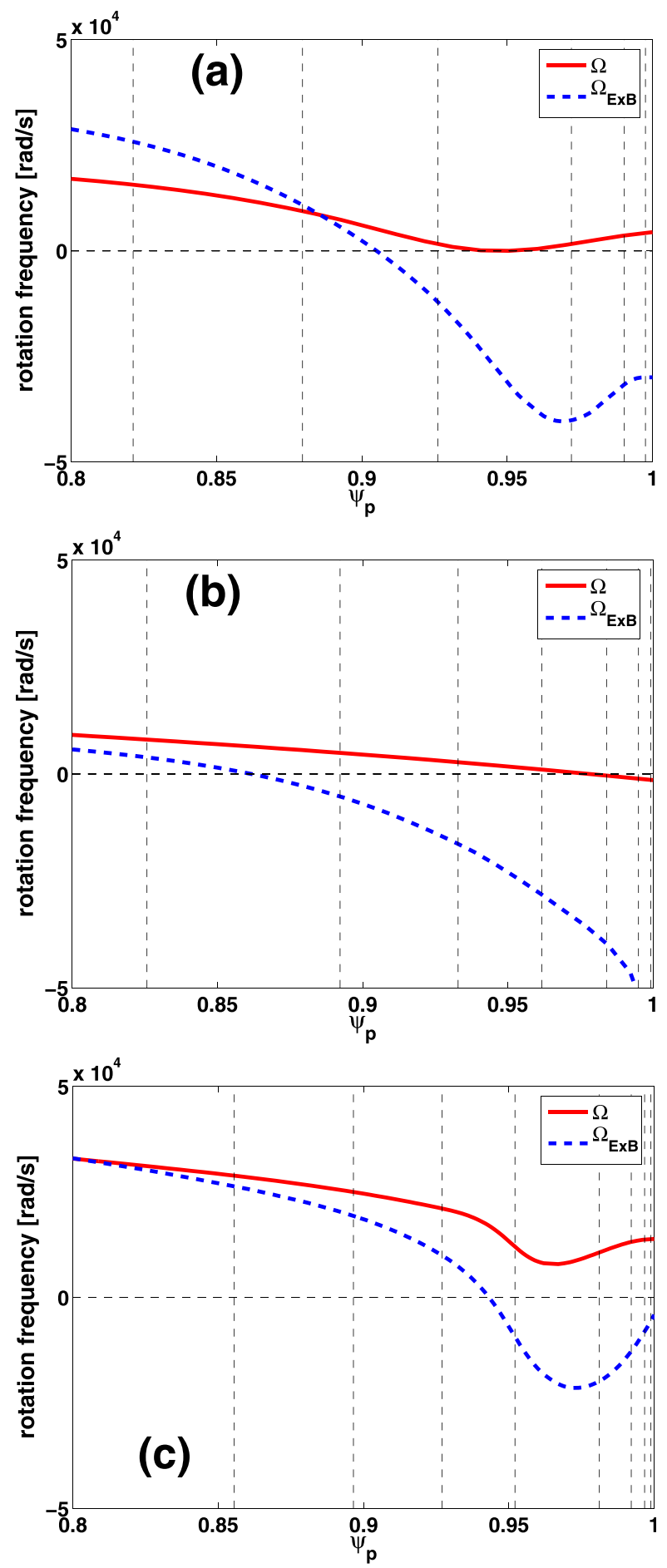

FIG. 9. The reconstructed (from the measurements) plasma fluid toroidal rotation frequency $\Omega$ (solid lines) and the $\mathbf{E} \times \mathbf{B}$ toroidal rotation frequency $\Omega_{\mathrm{E} \times \mathrm{B}}$ (dashed lines), for (a) the ASDEX Upgrade discharge 30835 at $3200 \mathrm{~ms}$, (b) the ASDEX Upgrade discharge 33133 at $3000 \mathrm{~ms}$, and (c) the DIII-D discharge 164277 at $2500 \mathrm{~ms}$. 

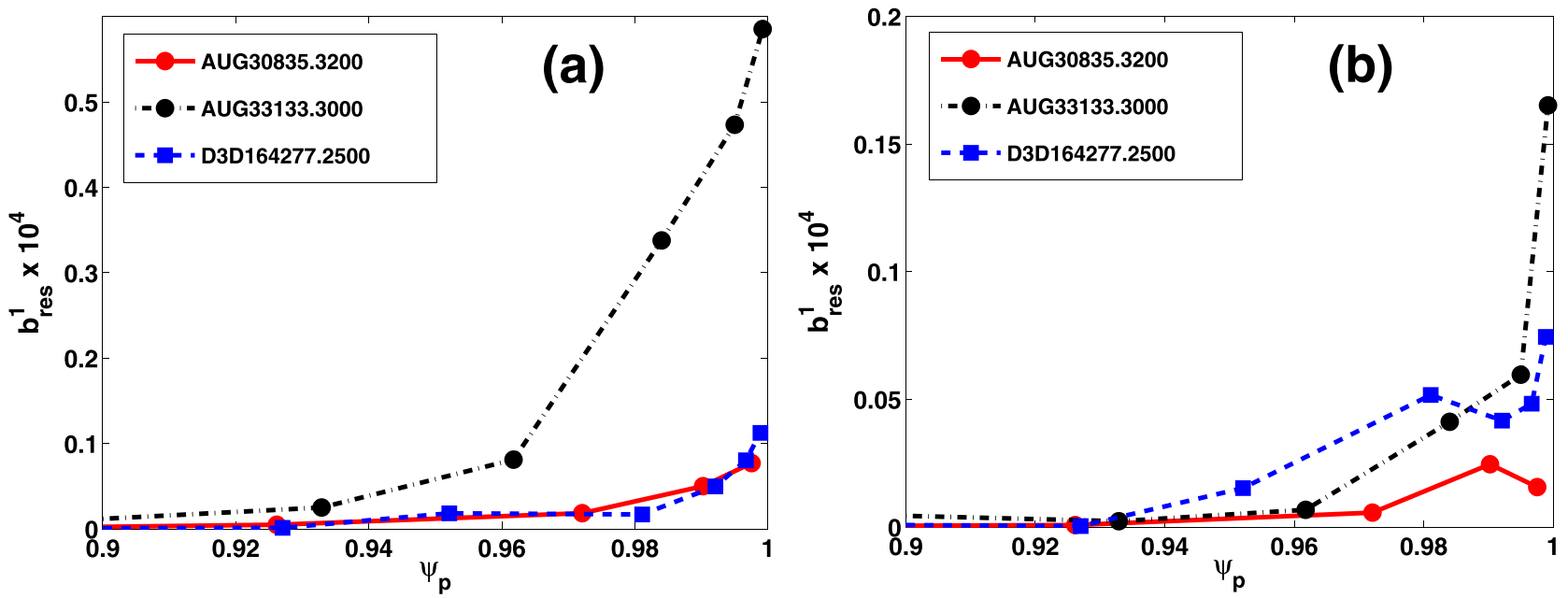

FIG. 10. Comparison of the computed plasma response, in terms of amplitude of the resonant radial field components near the plasma edge, among three discharges with different plasma shaping: the ASDEX Upgrade discharges 30835 and 33133, the DIII-D discharge 164277. Computations are performed assuming (a) the fluid rotation frequency $\Omega$, and (b) the $\mathbf{E} \times \mathbf{B}$ rotation frequency $\Omega_{\mathrm{E} \times \mathrm{B}}$, in the single fluid plasma response model.

with the angular frequency of $\Omega_{\mathrm{E} \times \mathrm{B}}$. These two rotation frequencies, for each of the three plasmas, are compared in the plasma edge region in Fig. 9. It is evident that the fluid and the $\mathbf{E} \times \mathbf{B}$ flow profiles are qualitatively different in these plasmas. In particular, the $\mathbf{E} \times \mathbf{B}$ flow speed reverses sign in the pedestal top region, in all three discharges. This may affect the flow screening of the applied RMP fields. This is also the main motivation for us to consider these two different flow models in this study.

Figure 10 compares the MARS-F computed plasma response, in terms of the resonant radial field component $b_{\text {res }}^{1}$, between three discharges with different plasma shaping, assuming the fluid flow model (a) and the $\mathbf{E} \times \mathbf{B}$ flow model (b), respectively. The fluid flow model does not distinguish between the ASDEX Upgrade low triangularity case (ELM mitigation) and the DIII-D ISS case (ELM suppression) in terms of $b_{\text {res. }}^{1}$. On the other hand, the fluid flow model yields a strong $b_{\text {res }}^{1}$ response for the high triangularity ASDEX Upgrade discharge 33133, at the rational surface $q=m /$ $n=8 / 2$ (near $\psi_{p}=0.96$ ). This is largely due to the fact that

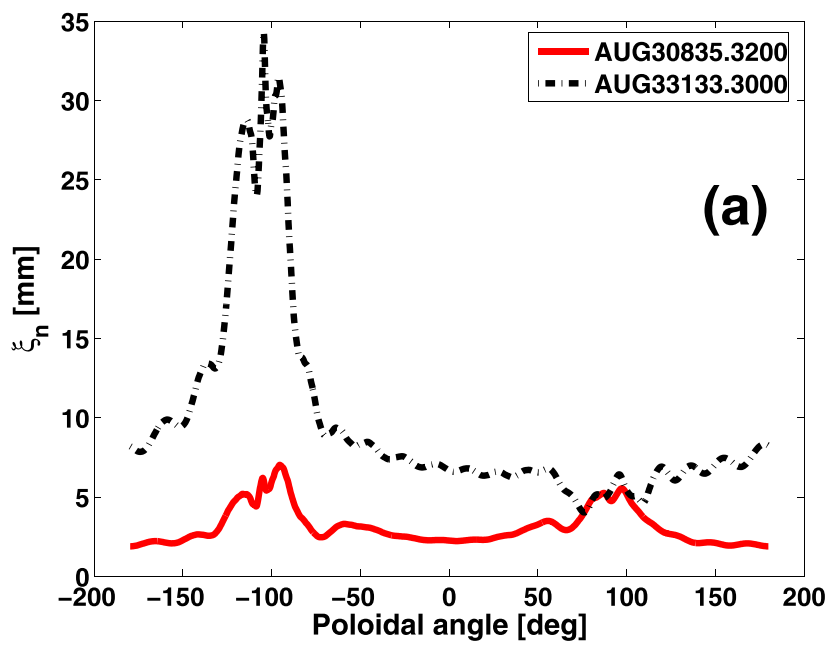

the fluid rotation frequency nearly vanishes near this rational surface, as shown in Fig. 9(b). [We note that the fluid flow also nearly vanishes in discharge 30835 as shown in Fig. 9 (a), but not near a rational surface (in fact between $7 / 2$ and $8 / 2$ surfaces). This does not yield an amplified response of the resonant field harmonic.]

Assuming the $\mathbf{E} \times \mathbf{B}$ flow model, Fig. 10(b) shows that the $b_{\text {res }}^{1}$ response near the plasma edge (in the pedestal region) is generally stronger in the ELM suppressed cases (both ASDEX Upgrade and DIII-D) than that in the ELM mitigation case (ASDEX Upgrade 30835). It remains, however, to clarify whether this larger response directly comes from the difference in the plasma shaping. This will be examined later on in this work.

The other figure of merit, which can be used to measure the plasma response, is the plasma surface displacement. ${ }^{19}$ The plasma surface displacements, as functions of the poloidal angle, are plotted and compared in Fig. 11 for the two ASDEX Upgrade equilibria with low and high upper triangularity shaping. Since the displacement is a dimensional

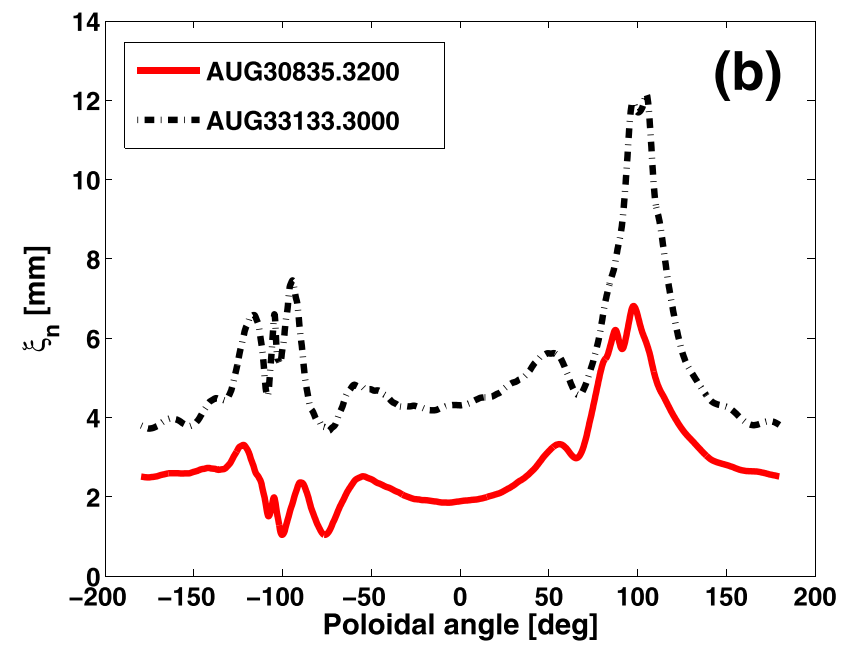

FIG. 11. Comparison of the computed plasma surface displacement amplitude along the poloidal angle, between the ELM mitigated case (30835) and the suppressed case (33133) in ASDEX Upgrade. Computations are performed assuming (a) the fluid rotation frequency $\Omega$, and (b) the $\mathbf{E} \times \mathbf{B}$ rotation frequency $\Omega_{\mathrm{E} \times \mathrm{B}}$, in the single fluid plasma response model. The outboard midplane corresponds to the poloidal angle of about 0 , and the X-point about $-100^{\circ}$. 
quantity, it is reasonable to perform the comparison within the same device. Again both the fluid flow and the $\mathbf{E} \times \mathbf{B}$ flow models are considered, with results reported in Figs. 11(a) and 11(b), respectively. A general observation is that the RMP fields induce larger plasma displacements in the ASDEX Upgrade plasma with stronger shaping. In particular, with the $\mathbf{E} \times \mathbf{B}$ flow, the plasma displacement near the $\mathrm{X}$-point is significantly larger with the high-triangularity shaping, where the ELM suppression has been achieved in experiments, as compared to the low triangularity shaping, where only ELM mitigation has been obtained.

We have also performed plasma response computations using an MHD-kinetic hybrid formulation (the MARS-K model ${ }^{35}$ ), where the precessional drift resonance of both bulk thermal ions and electrons is taken into account. The drift kinetic response from thermal particles is found to be almost identical to what have been shown in Figs. 10 and 11, indicating that the kinetic effect is weak in these modelled plasmas, in terms of the plasma response.

The stronger plasma response for the ELM suppressed cases (both ASDEX Upgrade and DIII-D), as shown in Figs. 10 and 11 , may be contributed from several factors. One obvious candidate is the plasma shaping. In order to better quantify this factor, we choose one equilibrium as the basis-in this study the one from ASDEX Upgrade 30835 at $3200 \mathrm{~ms}-$ and gradually vary the plasma shape while keeping the other radial profiles (pressure, current density, and toroidal flow) unchanged. We introduce a parametric shaping

$$
\begin{aligned}
S(\alpha)= & (1-\alpha)(1-2 \alpha) S_{30835}+4 \alpha(1-\alpha) S_{33133} \\
& +\alpha(2 \alpha-1) S_{164277},
\end{aligned}
$$

where $S_{30835}$ and $S_{33133}$ are the plasma boundary shapes from the ASDEX Upgrade discharges 30835 and 33133, respectively, as shown in Fig. 7 (a). $S_{164277}$ is the plasma shape from DIII-D discharge 164277 as shown in Fig. 7(a), but with the plasma minor radius shrunk to match that of the ASDEX Upgrade plasmas. The new shape $S(\alpha)$ is then introduced with a shaping factor $\alpha$, such that at $\alpha=0,0.5$, and 1 , the shapes $S_{30835}, S_{33133}$, and $S_{164277}$ are recovered, respectively. Figure 12(a) shows eleven examples of the plasma shapes, corresponding to $\alpha=0,0.1,0.2, \ldots, 1$.
It is not possible to obtain self-consistent toroidal equilibria with the variation of the plasma shape alone. In this study, we allow the total plasma current to vary, but keeping the edge safety factor the same as that of base equilibrium from ASDEX Upgrade discharge 30825, i.e., we keep $q_{95}=3.760$ while performing the shaping scan. The resulting plasma current variation, as a function of the shaping factor $\alpha$, is shown in Fig. 12(b).

Figures 13 and 14 report the main results of the MARS-F computed plasma response, from the aforementioned shaping scan. There is a certain trend of increasing the plasma response near the $q=m / n=8 / 2$ surface $\left(\psi_{p} \sim 0.97\right.$ in Fig. 13(b)), but the effect is moderate. We should note that, by assuming the same amount of plasma current in the RMP coils, the vacuum resonant field amplitude generally decreases with increasing plasma shaping. Therefore, the "effective" increase of the plasma response would be stronger with shaping, if we were matching the vacuum field component instead of the RMP current. The same holds for the plasma surface displacement (Fig. 14), which does not show significant change with shaping, by fixing the RMP coil current. Using different flow models (the fluid versus the $\mathbf{E} \times \mathbf{B}$ flow models in Figs. 14(a) and 14(b), respectively) does not change the conclusion here.

The above study thus confirms that the plasma shape variation alone cannot explain the larger plasma response computed for the ELM suppressed cases. The other factors, such as the plasma (pedestal) pressure and current density, may also play a role. It is known from the previous studies that the plasma kink response increases with pressure ${ }^{36,37}$ or parallel edge current. ${ }^{38}$

Work has also recently been initiated for modelling the ELM suppression experiments in KSTAR. KSTAR is so far the only device that can be used to study the ELM control with a coil system that is similar to the ITER design (i.e., three rows of in-vessel coils). Figure 15 shows a typical plasma shape in KSTAR, together with the RMP coil geometry. ${ }^{39}$ Because there are three rows of coils located at the low field side of the torus, KSTAR, as well as ITER, has more flexibility in choosing the poloidal spectrum of the applied field, by tuning the relative coil phasing between three rows.

One example is shown in Fig. 16, where we fix the toroidal phase $\Phi^{M}$ of the middle row coil currents and
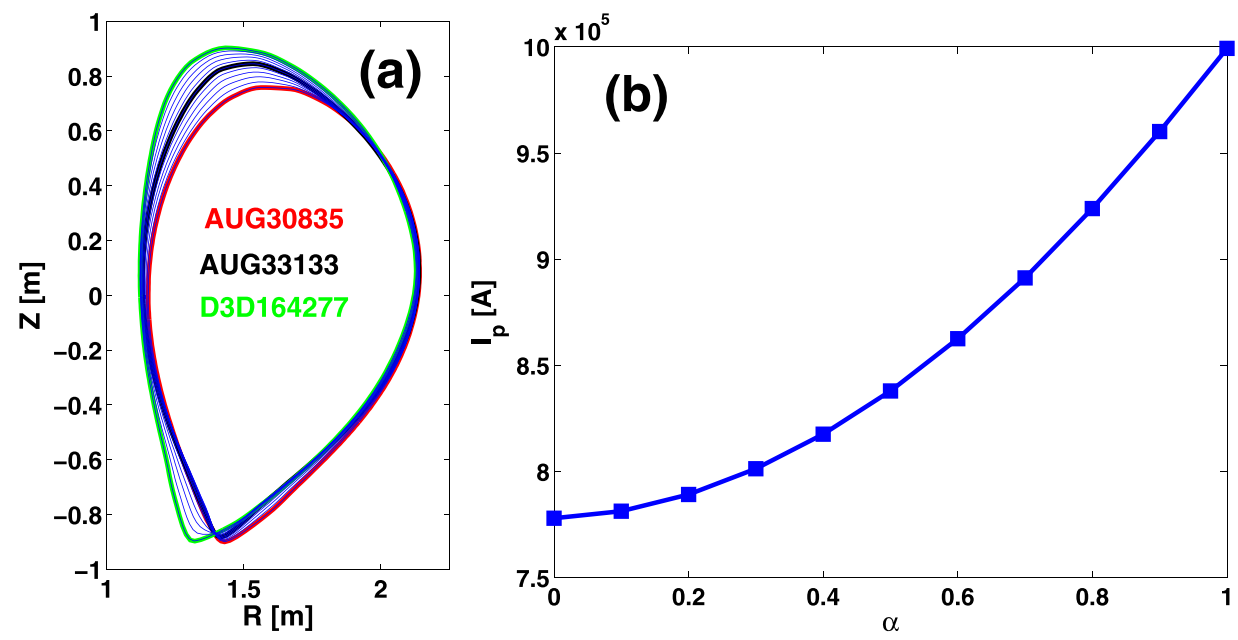

FIG. 12. (a) A systematic variation of the plasma boundary shape, introduced by a scaling parameter $\alpha$ and covering those of ASDEX Upgrade discharges $30835(\alpha=0)$ and $33133(\alpha=0.5)$, as well as the "shrunk" version of the DIII-D discharge $164277(\alpha=1)$. The equilibrium radial profiles for the pressure and the (surface averaged) toroidal current density are fixed, being the same as that from ASDEX Upgrade discharges 30835 . With fixed toroidal field and fixed $q_{95}$ value during the shaping scan, the self-consistently computed equilibrium solutions result in varying total plasma current plotted in (b). 

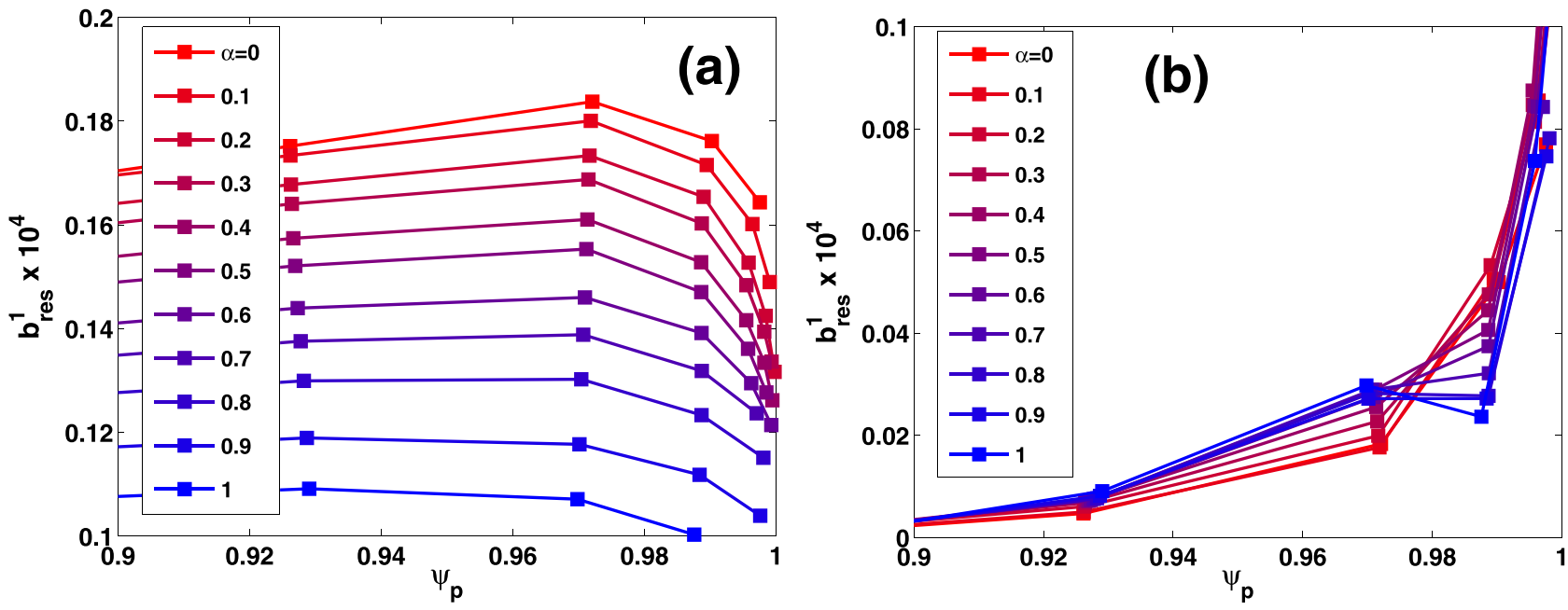

FIG. 13. Comparison of the amplitude of the resonant radial field components near the plasma edge, for (a) the vacuum field and (b) the total response field, while scanning the plasma boundary shape as shown in Fig. 12. The RMP coil current is kept unchanged during the shaping scan. Considered is the $n=2$ coil current configuration with $90^{\circ}$ coil phasing. The fluid rotation is assumed for the plasma response computations.
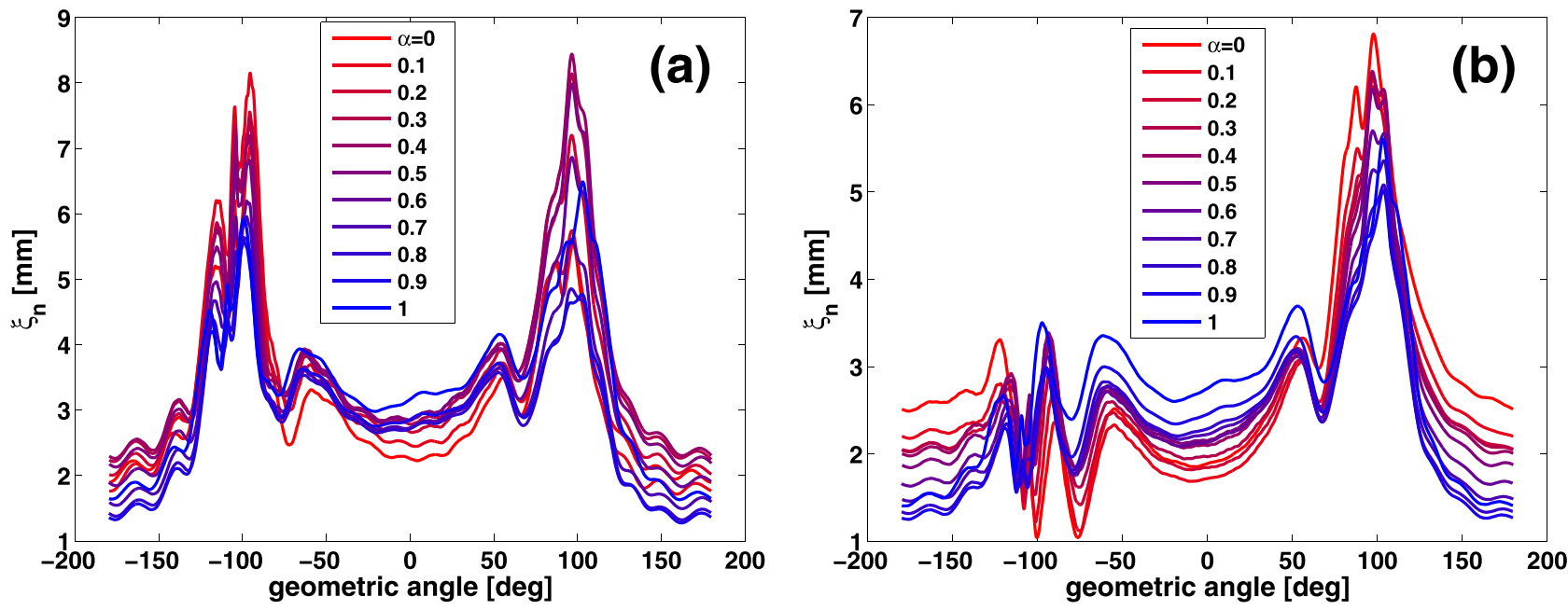

FIG. 14. The variation of the computed plasma surface displacement amplitude, along the poloidal angle, with varying plasma boundary shape as shown in Fig. 12. The plasma response is computed assuming (a) the fluid rotation model and (b) the $\mathbf{E} \times \mathbf{B}$ rotation model.
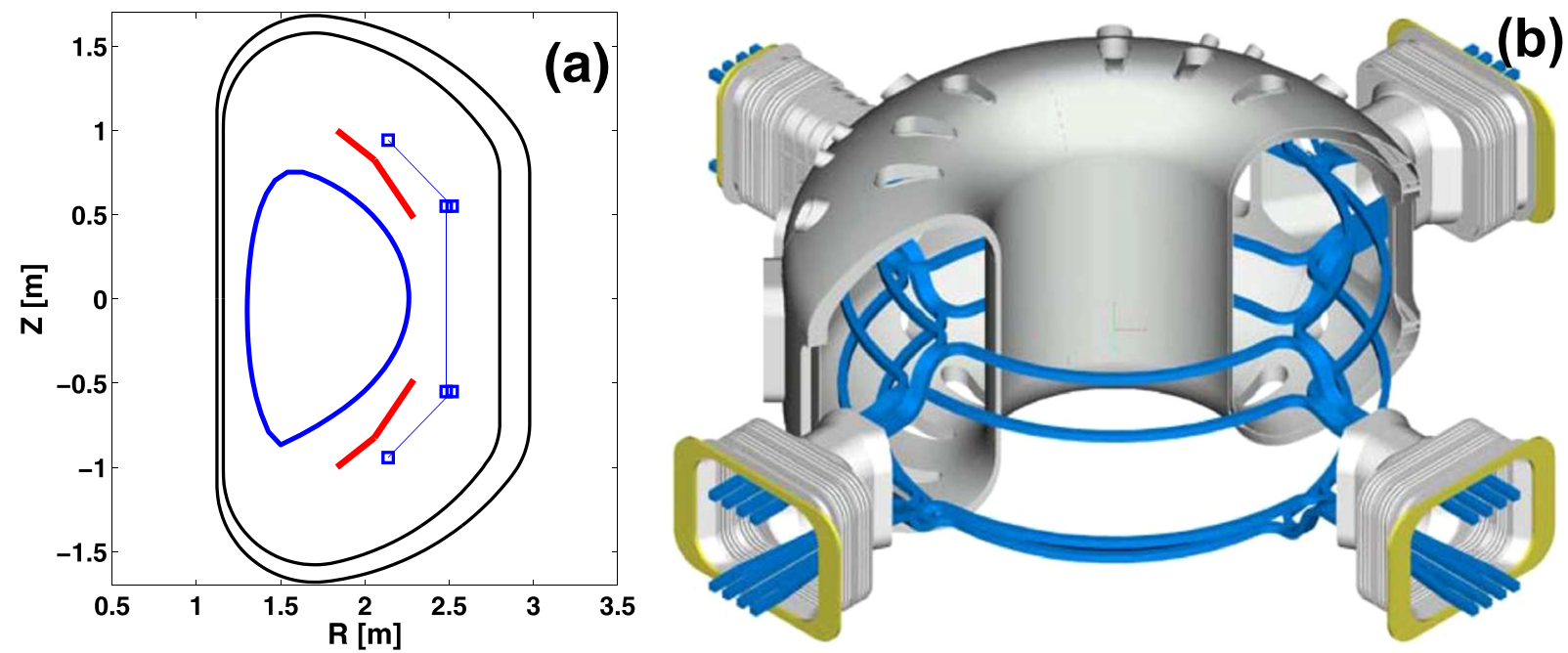

FIG. 15. The ELM control coil geometry in KSTAR, plotted (a) (in blue) together with the plasma boundary shape (based on discharge 11341 at $7 \mathrm{~s}$ ), the KSTAR double-wall vacuum vessel (n black), and the two sets (upper and lower, in red) of passive stabilizing plates, and (b) in a 3D view. 


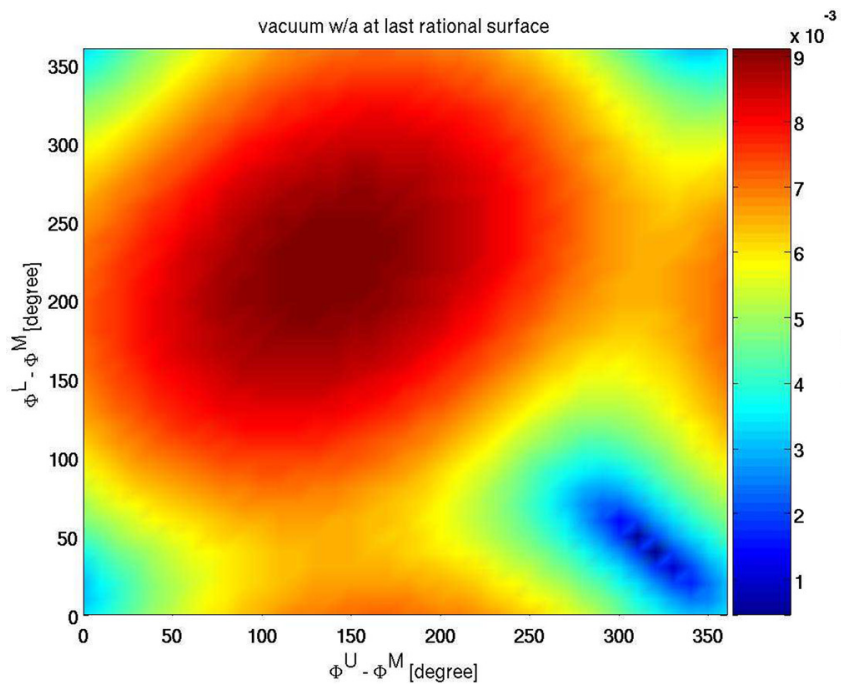

(a)

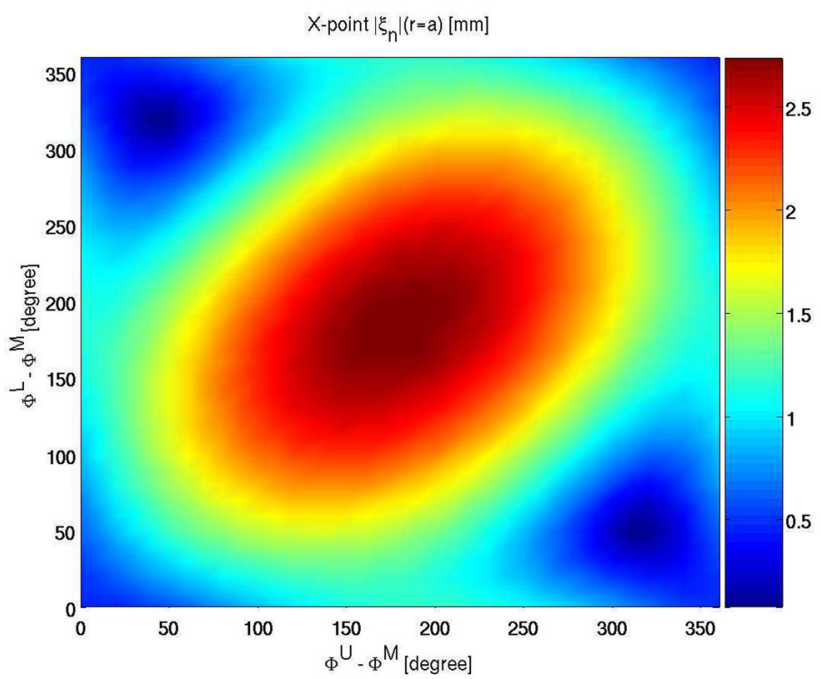

(c)

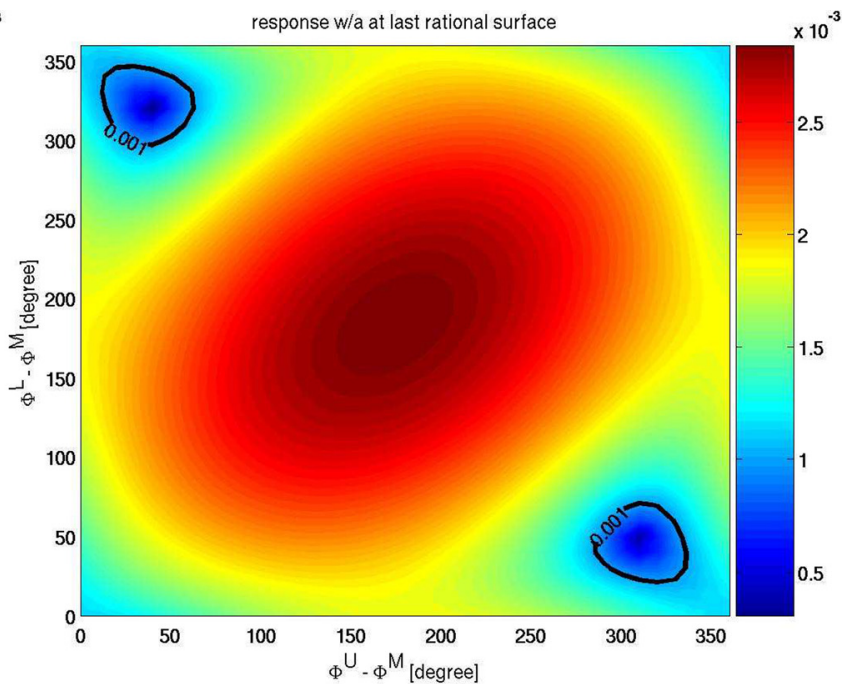

(b)

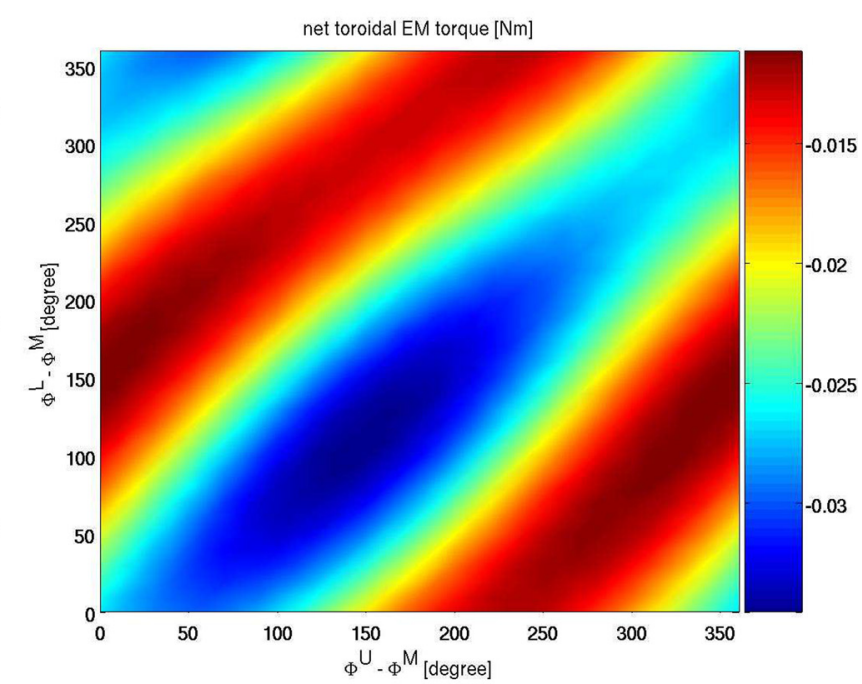

(d)

FIG. 16. The computed (a) vacuum island width near the plasma edge, (b) island width including resistive plasma response, (c) the plasma surface displacement near the X-point, and (d) the net electromagnetic torque inside the plasma, while varying the relative coil phasing between the upper and lower rows of coils in KSTAR, with respect to the middle row. All three rows of coils are assumed to be in the $n=2$ configuration, with the coil current of 1 kAt. Computations are based on the KSTAR equilibrium from discharge 11341 at $7 \mathrm{~s}$.

independently vary the coil current phase for the upper $\left(\Phi^{U}\right)$ and lower $\left(\Phi^{L}\right)$ rows. The computations are performed assuming the $n=2$ coil configuration, as in the KSTAR discharge 11341. The modelled plasma has the on-axis safety factor $q_{0}=1.05$ and the edge safety factor $q_{95}=3.64$. The normalized plasma pressure is $\beta_{N}=2.50$. The computed plasma response, quantified in terms of the magnetic island width near the plasma edge, the plasma displacement near the X-point, and the net electromagnetic torque inside the plasma, is shown in Figs. 16(b)-16(d), respectively. A clear optimum, of $\Phi^{U}-\Phi^{M} \simeq \Phi^{L}-\Phi^{M} \simeq 180^{\circ}$, is predicted, which yields the largest plasma response as shown in Figs. 16(b) and 16(c). This essentially corresponds to the odd parity configuration between the middle-row and off-middle rows of coils. Interestingly, both figures of merit-the resonant radial field amplitude (b) and the plasma X-point displacement (c) - yield the same optimum for the coil phasing. The vacuum field (a) yields a different optimum. On the other hand, the optimal coil phasing, which maximizes the plasma response, also leads to large (not the largest though) electromagnetic torque that can potentially brake the plasma flow. Therefore, in reality, the best choice of the coil phasing may have to be a compromise between maximizing the plasma response on one hand and minimizing the flow damping on the other hand.

We mention that a direct comparison of the above modelling results with KSTAR experiments, as well as systematic modelling of the KSTAR ELM suppression experiments with more relevant plasma equilibria, remains to be a future work.

\section{ON THE ROLE OF EDGE-PEELING PLASMA RESPONSE}

Extensive multi-machine modelling efforts on the ELM mitigation, as well as so far limited efforts on the ELM suppression, all point to the important role played by the edge-peeling plasma response. The applicability of the 
TABLE II. Applicability of the edge-peeling criterion to ELM control experiments. Symbol " " indicates successful application so far (with references where applicable), for the scanned parameters listed in the table.

\begin{tabular}{|c|c|c|c|c|c|c|c|}
\hline Device & $R_{0}(\mathrm{~m})$ & $R_{0} / a$ & \#Rows $\times$ \#coils & $\mathrm{nn}$ & Scanned parameters & Mitigation & Suppression \\
\hline MAST & 0.9 & 1.7 & $6+12$ & $3(1,2,4,6)$ & $\Delta \Phi, q_{95}$ & $\sqrt{ }($ Ref. 7) & \\
\hline ASDEX & 1.7 & 3.3 & $2 \times 8$ & $2(1,3,4)$ & $\Delta \Phi, q_{95}, \beta_{N}$ & $\sqrt{ }($ Refs. 4 and 10-13) & $\sqrt{ }$ \\
\hline \multicolumn{8}{|l|}{ Upgrade } \\
\hline DIII-D & 1.65 & 2.8 & $2 \times 6$ & $3(1,2)$ & $\Delta \Phi, q_{95}$ & & $\checkmark$ \\
\hline EAST & 1.85 & 4.3 & $2 \times 8$ & $1,2,4$ & $\Delta \Phi$ & $\sqrt{ }($ Ref. 14) & $\sqrt{ }($ Refs. 3 and 14$)$ \\
\hline JET & 2.9 & 3.1 & $1 \times 4$ & 1,2 & $\beta_{N}, q_{95}$ & $\sqrt{ }($ Ref. 34) & \\
\hline KSTAR & 1.8 & 3.7 & $3 \times 4$ & 1,2 & $\Delta \Phi$ & & \\
\hline HL-2A & 1.65 & 4.4 & $2 \times 2$ & $1,3,5, \ldots$ & $\Delta \Phi, q_{95}$ & $\sqrt{ }$ & \\
\hline
\end{tabular}

edge-peeling criterion to multiple devices is summarized in Table II, where the basic machine parameters and available coil configuration in each machine are also listed and compared. Various parameter scans - mostly the coil phasing $\Delta \Phi$ and the edge safety factor $q_{95}$ scans-have been performed for a given device. As a peculiar point, we note that MAST had 6 coils in the upper row and 12 coils in the lower row, which allows the RMP spectrum up to $n=6$, using the lower row alone.

We emphasize that such a comparative cross-machine investigation, though valuable in identifying the key plasma response metric for ELM control, is not sufficient in understanding the ELM suppression physics. More study, probably with enhanced plasma models beyond macroscopic MHD, may be essential to identify the key physics differences between the ELM mitigation and suppression.

\section{CONCLUSION AND DISCUSSION}

Quantitative toroidal modelling of the plasma response for multi-machine ELM control experiments, utilizing RMP fields, leads to a reasonable solid conclusion that the edgepeeling plasma response plays a significant role in ELM mitigation. In direct toroidal computations, the edge-peeling response often manifests itself as a large resonant radial field component near the plasma edge, or a strong plasma surface displacement near the X-point point in a divertor plasma. A closer analysis reveals that the large $\mathrm{X}$-point displacement is a sufficient but not a necessary condition when the edgepeeling response is dominant-a more general condition is the weak equilibrium poloidal field along the certain poloidal angle of the plasma surface. ${ }^{19}$ The edge-peeling response criterion is particularly useful in providing guidance for optimization of the RMP coil phasing, as has been demonstrated in MAST, ASDEX Upgrade, and EAST in the previous work, and in the detailed modelling for HL-2A in this study. Extensive modeling has also shown that the optimal coil phasing depends on the plasma conditions, in particular, on the edge- $q$ value $\left(q_{95}\right)$ as well as on the plasma pressure.

To achieve better ELM mitigation, while maximizing the edge-peeling response is essential, minimizing the corekink response may also be important to avoid undesired side effects (such as core flow damping or H-to-L back transition) on the plasma. For plasma-coil configurations where both the edge-peeling and the core-kink response can be simultaneously large, the best strategy seems to be maximizing the ratio of the plasma X-point to the outboard mid-plane displacement. This is so far the case for MAST and HL-2A plasmas. In fact, this displacement ratio serves as a good indicator of the experimentally observed ELM mitigation window in $q_{95}$ in HL-2A, as shown in this study. On the other hand, the relationship between the fluid model predicted X-point displacement and the lobe structures observed in experiments, ${ }^{40,41}$ near the X-point, needs further investigation.

The edge-peeling response criterion also helps to identify the best coil phasing for ELM suppression, as shown by the modeling results in EAST and DIII-D. More validation work, however, is needed for further confirmation, e.g., for ASDEX Upgrade and KSTAR plasmas.

It is, however, a more subtle issue to distinguish ELM mitigation and suppression from the modelling point of view, based on the macroscopic plasma response. Attempts made in this study, for the ASDEX Upgrade and DIII-D plasmas, show that the plasma response is generally stronger for the ELM suppressed cases, as compared to that of the ELM mitigated cases. This is partially related to the (stronger) plasma shaping. But the other plasma conditions, such as the (higher) pedestal pressure and/or current density, may also play a role. In real experiments, these factors are often coupled and perhaps act in a synergistic manner to help achieving ELM suppression.

The macroscopic plasma response, based on linear fluid (or two-fluid), ideal, or resistive MHD models, helps to capture some of the physics associated with the ELM control, but certainly cannot answer all the questions. In particular, it is not clear whether these kinds of models can fully identify the ELM suppression physics. The role of large magnetic islands near the pedestal top ${ }^{42}$ on the field screening needs to be further investigated, based on improved linear MHD models, non-linear models, or even kinetic models. The drift kinetic effect from bulk thermal particles, however, has been found weak in modifying the plasma response, for the ASDEX Upgrade and DIII-D plasmas considered in this work. Other physics mechanisms such as turbulence, which helps to enhance the pedestal transport during the ELM suppression, may also need to be considered in the future.

Another important question, which has so far not been systematically addressed in the plasma response based modelling, is the (minimal) RMP current requirement, in order to achieve the desired ELM control. This is a critical 
issue for ITER and for any other future devices where ELM control is required. A semi-empirical approach, combining the experimental observations with the computed plasma response, may help to identify such requirements. First principle based approaches, as it appears, are still not sufficiently mature to directly address this question, but efforts should certainly be devoted here.

\section{ACKNOWLEDGMENTS}

This work has been carried out within the framework of the EUROfusion Consortium and has received funding from the Euratom research and training programme 2014-2018 under Grant Agreement No. 633053 and from the RCUK Energy Programme [Grant No. EP/I501045]. Work is also part funded by National Natural Science Foundation of China (NSFC) [Grant Nos. 11428512 and 11405029] and by National Magnetic Confinement Fusion Science Program under Grant No. 2015GB104004. The views and opinions expressed herein do not necessarily reflect those of the European Commission.

${ }^{1}$ T. E. Evans, R. A. Moyer, P. R. Thomas, J. G. Watkins, T. H. Osborne, J. A. Boedo, E. J. Doyle, M. E. Fenstermacher, K. H. Finken, R. J. Groebner, M. Groth, J. H. Harris, R. J. La Haye, C. J. Lasnier, S. Masuzaki, N. Ohyabu, D. G. Pretty, T. L. Rhodes, H. Reimerdes, D. L. Rudakov, M. J. Schaffer, G. Wang, and L. Zeng, Phys. Rev. Lett. 92, 235003 (2004).

${ }^{2}$ Y. M. Jeon, J.-K. Park, S. W. Yoon, W. H. Ko, S. G. Lee, K. D. Lee, G. S. Yun, Y. U. Nam, W. C. Kim, J.-G. Kwak, K. S. Lee, H. K. Kim, and H. L. Yang (KSTAR team), Phys. Rev. Lett. 109, 035004 (2012).

${ }^{3}$ Y. Sun, Y. Liang, Y. Q. Liu, S. Gu, X. Yang, W. Guo, T. Shi, M. Jia, L. Wang, B. Lyu, C. Zhou, A. Liu, Q. Zang, H. Liu, N. Chu, H. H. Wang, T. Zhang, J. Qian, L. Xu, K. He, D. Chen, B. Shen, X. Gong, X. Ji, S. Wang, M. Qi, Y. Song, Q. Yuan, Z. Sheng, G. Gao, P. Fu, and B. Wan, Phys. Rev. Lett. 117, 115001 (2016).

${ }^{4}$ W. Suttrop, A. Kirk, R. Nazikian, N. Leuthold, E. Strumberger, M. Willensdorfer, M. Cavedon, M. Dunne, R. Fischer, S. Fietz, J. C. Fuchs, Y. Q. Liu, R. M. McDermott, F. Orain, D. A. Ryan, E. Viezzer, the ASDEX Upgrade Team, the DIII-D Team, and the Eurofusion MST1 Team, Plasma Phys. Control. Fusion 59, 014049 (2017).

${ }^{5}$ Y. Liang, H. R. Koslowski, P. R. Thomas, E. Nardon, B. Alper, P. Andrew, Y. Andrew, G. Arnoux, Y. Baranov, M. Bécoulet, M. Beurskens, T. Biewer, M. Bigi, K. Crombe, E. De La Luna, P. de Vries, W. Fundamenski, S. Gerasimov, C. Giroud, M. P. Gryaznevich, N. Hawkes, S. Hotchin, D. Howell, S. Jachmich, V. Kiptily, L. Moreira, V. Parail, S. D. Pinches, E. Rachlew, and O. Zimmermann, Phys. Rev. Lett. 98, 265004 (2007).

${ }^{6}$ A. Kirk, E. Nardon, R. Akers, M. Bécoulet, G. De Temmerman, B. Dudson, B. Hnat, Y. Q. Liu, R. Martin, P. Tamain, D. Taylor, and the MAST team, Nucl. Fusion 50, 034008 (2010).

${ }^{7}$ Y. Q. Liu, A. Kirk, Y. Gribov, M. P. Gryaznevich, T. C. Hender, and E. Nardon, Nucl. Fusion 51, 083002 (2011).

${ }^{8}$ N. M. Ferraro, Phys. Plasmas 19, 056105 (2012).

${ }^{9}$ S. R. Haskey, M. J. Lanctot, Y. Q. Liu, J. M. Hanson, B. D. Blackwell, and R. Nazikian, Plasma Phys. Controlled Fusion 56, 035005 (2014).

${ }^{10}$ D. A. Ryan, Y. Q. Liu, A. Kirk, W. Suttrop, B. Dudson, M. Dunne, R. Fischer, J. C. Fuchs, M. Garcia-Munoz, B. Kurzan, P. Piovesan, M. Reinke, M. Willensdorfer, the ASDEX-Upgrade team, and the EUROfusion MST1 team, Plasma Phys. Controlled Fusion 57, 095008 (2015).

${ }^{11}$ Y. Q. Liu, D. Ryan, A. Kirk, L. Li, W. Suttrop, M. Dunne, R. Fischer, J. C. Fuchs, B. Kurzan, P. Piovesan, M. Willensdorfer, the ASDEX Upgrade Team, and the EUROfusion MST1 Team, Nucl. Fusion 56, 056015 (2016)

${ }^{12}$ L. Li, Y. Q. Liu, A. Kirk, N. Wang, Y. Liang, D. Ryan, W. Suttrop, M. Dunne, R. Fischer, J. C. Fuchs, B. Kurzan, P. Piovesan, M. Willensdorfer, F. C. Zhong, the ASDEX Upgrade Team and the EUROfusion MST1 Team, Nucl. Fusion 56, 126007 (2016).
${ }^{13}$ F. Orain, M. Hoelzl, E. Viezzer, M. Dunne, M. Bécoulet, G. T. A. Huijsmans, P. Cahyna, J. Morales, M. Willensdorfer, and W. Suttrop, Nucl. Fusion 57, 022013 (2017).

${ }^{14}$ X. Yang, Y. Sun, Y. Liu, S. Gu, Y. Liu, H. Wang, L. Zhou, and W. Guo, Plasma Phys. Controlled Fusion 58, 114006 (2016).

${ }^{15}$ F. Orain, M. Bécoulet, G. Dif-Pradalier, G. Huijsmans, S. Pamela, E. Nardon, C. Passeron, G. Latu, V. Grandgirard, A. Fil, A. Ratnani, I. Chapman, A. Kirk, A. Thornton, M. Hoelzl, and P. Cahyna, Phys. Plasmas 20, 102510 (2013).

${ }^{16}$ Y. Q. Liu, R. Akers, I. T. Chapman, Y. Gribov, G. Z. Hao, G. T. A. Huijsmans, A. Kirk, A. Loarte, S. D. Pinches, M. Reinke, D. Ryan, Y. Sun, and Z. R. Wang, Nucl. Fusion 55, 063027 (2015).

${ }^{17}$ L. Zhou, Y. Liu, Y. Liu, and X. Yang, Plasma Phys. Controlled Fusion 58, 115003 (2016).

${ }^{18}$ G. T. A. Huijsmans, C. S. Chang, C. S. N. Ferraro, L. Sugiyama, F. Waelbroeck, X. Q. Xu, A. Loarte, and S. Futatani, Phys. Plasmas 22, 021805 (2015).

${ }^{19}$ Y. Q. Liu, C. J. Ham, A. Kirk, L. Li, A. Loarte, D. A. Ryan, Y. Sun, W. Suttrop, X. Yang, and L. N. Zhou, Plasma Phys. Controlled Fusion 58 , 114005 (2016)

${ }^{20}$ M. J. Lanctot, H. Reimerdes, A. M. Garofalo, M. S. Chu, J. M. Hanson, Y. Q. Liu, G. A. Navratil, I. N. Bogatu, Y. In, G. L. Jackson, R. J. La Haye, M. Okayabashi, J.-K. Park, M. J. Schaffer, O. Schmitz, E. J. Strait, and A. D. Turnbull, Phys. Plasmas 18, 056121 (2011).

${ }^{21}$ A. D. Turnbull, N. M. Ferraro, V. A. Izzo, E. A. Lazarus, J.-K. Park, W. A. Cooper, S. P. Hirshman, L. L. Lao, M. J. Lanctot, S. Lazerson, Y. Q. Liu, A. Reiman, and F. Turco, Phys. Plasmas 20, 056114 (2013).

${ }^{22}$ Y. Q. Liu, A. Kirk, Y. Sun, P. Cahyna, I. T. Chapman, P. Denner, G. Fishpool, A. M. Garofalo, J. R. Harrison, E. Nardon, and the MAST Team, Plasma Phys. Controlled Fusion 54, 124013 (2012).

${ }^{23}$ R. E. Waltz and N. M. Ferraro, Phys. Plasmas 22, 042507 (2015).

${ }^{24}$ J. Varje, O. Asunta, M. Cavinato, M. Gagliardi, E. Hirvijoki, T. Koskela, T. Kurki-Suonio, Y. Q. Liu, V. Parail, G. Saibene, S. Sipilä, A. Snicker, K. Särkimäki, and S. Äkäslompolo, Nucl. Fusion 56, 046014 (2016).

${ }^{25}$ C. Paz-Soldan, R. Nazikian, S. R. Haskey, N. C. Logan, E. J. Strait, N. M. Ferraro, J. M. Hanson, J. D. King, M. J. Lanctot, R. A. Moyer, M. Okabayashi, J.-K. Park, M. W. Shafer, and B. J. Tobias, Phys. Rev. Lett. 114, 105001 (2015).

${ }^{26}$ Y. Q. Liu, A. Bondeson, C. M. Fransson, B. Lennartson, and C. Breitholtz, Phys. Plasmas 7, 3681 (2000).

${ }^{27}$ Y. Q. Liu, A. Kirk, and E. Nardon, Phys. Plasmas 17, 122502 (2010).

${ }^{28}$ Y. Q. Liu, A. Kirk, A. J. Thornton, and The MAST Team, Plasma Phys. Controlled Fusion 56, 104002 (2014).

${ }^{29}$ M. F. Heyn, I. B. Ivanov, S. V. Kasilov, W. Kernbichler, I. Joseph, R. A. Moyer, and A. M. Runov, Nucl. Fusion 48, 024005 (2008).

${ }^{30}$ M. Bécoulet, G. Huysmans, X. Garbet, E. Nardon, D. Howell, A. Garofalo, M. Schaffer, T. Evans, K. Shaing, A. Cole, J.-K. Park, and P. Cahyna, Nucl. Fusion 49, 085011 (2009).

${ }^{31}$ F. L. Waelbroeck, I. Joseph, E. Nardon, M. Bécoulet, and R. Fitzpatrick, Nucl. Fusion 52, 074004 (2012).

${ }^{32}$ I. T. Chapman, A. Kirk, R. J. Akers, C. J. Ham, J. R. Harrison, J. Hawke, Y. Q. Liu, K. G. McClements, S. Pamela, S. Saarelma, R. Scannell, A. J. Thornton, and The MAST Team, Nucl. Fusion 54, 123003 (2014).

${ }^{33}$ A. Kirk, W. Suttrop, I. T. Chapman, Y. Q. Liu, R. Scannell, A. J. Thornton, L. Barrera Orte, P. Cahyna, T. Eich, R. Fischer, C. Fuchs, C. Ham, J. R. Harrison, M. W. Jakubowski, B. Kurzan, S. Pamela, M. Peterka, D. Ryan, S. Saarelma, B. Sieglin, M. Valovic, M. Willensdorfer, and MAST and ASDEX Upgrade Teams, Nucl. Fusion 55, 043011 (2015).

${ }^{34}$ Y. Q. Liu, S. Saarelma, M. P. Gryaznevich, T. C. Hender, D. F. Howell, and JET-EFDA contributors, Plasma Phys. Controlled Fusion 52, 045011 (2010).

${ }^{35}$ Y. Q. Liu, M. S. Chu, I. T. Chapman, and T. C. Hender, Phys. Plasmas 15, 112503 (2008).

${ }^{36}$ Z. R. Wang, M. J. Lanctot, Y. Q. Liu, J.-K. Park, and J. E. Menard, Phys. Rev. Lett. 114, 145005 (2015).

${ }^{37}$ D. A. Ryan, Y. Q. Liu, L. Li, A. Kirk, M. Dunne, B. Dudson, P. Piovesan, W. Suttrop, M. Willensdorfer, the ASDEX Upgrade team, and the EUROfusion MST1 team, Plasma Phys. Controlled Fusion 59, 024005 (2017).

${ }^{38}$ A. Wingen, N. M. Ferraro, M. W. Shafer, E. A. Unterberg, J. M. Canik, T. E. Evans, D. L. Hillis, S. P. Hirshman, S. K. Seal, P. B. Snyder, and A. C. Sontag, Plasma Phys. Controlled Fusion 57, 104006 (2015). 
${ }^{39}$ H. K. Kim, H. L. Yang, G. H. Kim, J.-Y. Kim, H. Jhang, J. S. Bak, and G. S. Lee, Fusion Eng. Des. 84, 1029 (2009).

${ }^{40}$ A. Kirk, J. Harrison, Y. Q. Liu, E. Nardon, I. T. Chapman, P. Denner, and the MAST team, Phys. Rev. Lett. 108, 255003 (2012).

${ }^{41}$ J. R. Harrison, A. Kirk, I. T. Chapman, P. Cahyna, Y. Q. Liu, E. Nardon, and A. J. Thornton, Nucl. Fusion 54, 064015 (2014).
${ }^{42}$ R. Nazikian, C. Paz-Soldan, J. D. Callen, J. S. deGrassie, D. Eldon, T. E. Evans, N. M. Ferraro, B. A. Grierson, R. J. Groebner, S. R. Haskey, C. C. Hegna, J. D. King, N. C. Logan, G. R. McKee, R. A. Moyer, M. Okabayashi, D. M. Orlov, T. H. Osborne, J.-K. Park, T. L. Rhodes, M. W. Shafer, P. B. Snyder, W. M. Solomon, E. J. Strait, and M. R. Wade, Phys. Rev. Lett. 114, 105002 (2015). 